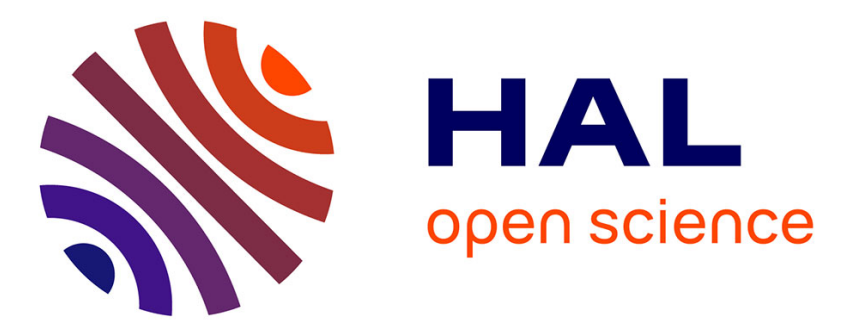

\title{
Flows of suspensions of particles in yield stress fluids
}

Guillaume Ovarlez, Fabien Mahaut, Stephanie Deboeuf, Nicolas Lenoir, Sarah

Hormozi, Xavier Chateau

\section{To cite this version:}

Guillaume Ovarlez, Fabien Mahaut, Stephanie Deboeuf, Nicolas Lenoir, Sarah Hormozi, et al.. Flows of suspensions of particles in yield stress fluids. Journal of Rheology, 2015, 59 (6), pp.1449. 10.1122/1.4934363 . hal-01223726

\section{HAL Id: hal-01223726 https://hal.science/hal-01223726}

Submitted on 10 Nov 2015

HAL is a multi-disciplinary open access archive for the deposit and dissemination of scientific research documents, whether they are published or not. The documents may come from teaching and research institutions in France or abroad, or from public or private research centers.
L'archive ouverte pluridisciplinaire HAL, est destinée au dépôt et à la diffusion de documents scientifiques de niveau recherche, publiés ou non, émanant des établissements d'enseignement et de recherche français ou étrangers, des laboratoires publics ou privés. 


\title{
Flows of suspensions of particles in yield stress fluids
}

\author{
Guillaume Ovarlez ${ }^{1,2 *}$,Fabien Mahaut ${ }^{2}$, Stéphanie Deboeuf ${ }^{2,3}$, \\ Nicolas Lenoir $^{2,4}$, Sarah Hormozi ${ }^{2,5}$, Xavier Chateau ${ }^{2}$ \\ 1 Univ. Bordeaux, CNRS, Solvay, LOF, UMR 5258, F-33608 Pessac, France \\ 2 Université Paris-Est, Laboratoire Navier (UMR 8205) \\ CNRS, ENPC, IFSTTAR, F-77420 Marne-la-Vallée, France \\ 3 Sorbonne Universités, UPMC Univ Paris 06, CNRS, UMR 7190 \\ Institut Jean Le Rond d'Alembert, F-75005 Paris, France \\ ${ }^{4}$ PLACAMAT, UMS 3626 CNRS-Univ. de Bordeaux, Pessac (France) \\ 5 Department of Mechanical Engineering, Ohio University \\ Athens, Ohio 45701-2979, USA
}

\begin{abstract}
Synopsis
We study the rheological behavior of suspensions of noncolloidal spheres in yield stress fluids (concentrated emulsions). These are good model systems for understanding, e.g., the rheology of fresh concrete or debris flows, and more generally the behavior of particles dispersed in any nonlinear material. We use magnetic resonance imaging (MRI) techniques to investigate the flows of these yield stress suspensions in a concentric-cylinder Couette geometry. We extend the theoretical approach of Chateau et al. (2008), valid for isotropic suspensions, to describe suspensions in simple shear flows, in which an anisotropic spatial distribution of particles is induced by flow. Theory and experiments show that the suspensions can be modeled by a Herschel-Bulkley behavior of same index as their interstitial fluid. We characterize the increase of their consistency and their yield stress with the particle volume fraction $\phi$ in the 0-50\% range. We observe a good agreement between the experimental variations of the consistency with $\phi$ and the theoretical prediction. This shows that the average apparent viscosity of the sheared interstitial material is correctly estimated and taken into account. We also observe shear-induced migration with similar properties as in a Newtonian fluid, which we predict theoretically, suggesting that particle normal stresses are proportional to the shear stress. However, the yield stress at flow stoppage increases much less than predicted. We also show that new features emerge in the rheology of the yield stress fluid when adding particles. We predict and observe the emergence of a nonzero normal stress difference at the yielding transition. We observe that the yield stress at flow start can differ from the yield stress at flow stoppage, and depends on flow history. It is likely a signature of a shear-dependent microstructure, due to the nonlinear behavior of the interstitial fluid, which makes these materials different from suspensions in Newtonian media. This is confirmed by direct characterization of shear-rate-dependent pair distribution functions using X-ray microtomography. This last observation explains why the theory predictions for the consistency can be correct while failing to model the yield stress at flow stoppage: a unique microstructure was indeed assumed as a first approximation. More sophisticated theories accounting for a shear-dependent microstructure are thus needed.
\end{abstract}

*e-mail: guillaume.ovarlez@u-bordeaux.fr 


\section{Contents}

I Introduction $\quad 2$

II Theory 5

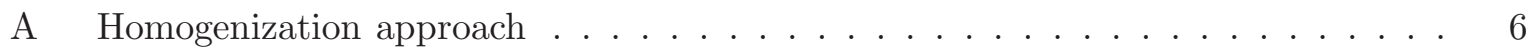

$1 \quad$ Choice of a local strain rate estimate . . . . . . . . . . . 6

2 Computation of the local strain rate estimate . . . . . . . . . . 6

3 From linear to nonlinear properties . . . . . . . . . . . . . 8

$4 \quad$ Application to yield stress fluids . . . . . . . . . . . . 8

$5 \quad$ Domain of validity . . . . . . . . . . . . . . . . . 9

B Phenomenological expressions . . . . . . . . . . . . . . . . 10

1 Isotropic suspensions . . . . . . . . . . . . . . . . . . . . . 10

$2 \quad$ Anisotropic suspensions . . . . . . . . . . . . . . . . . . 11

C Normal stress differences and shear-induced migration . . . . . . . . . . . . . 13

$\begin{array}{ll}\text { IIIMaterials and methods } & 14\end{array}$

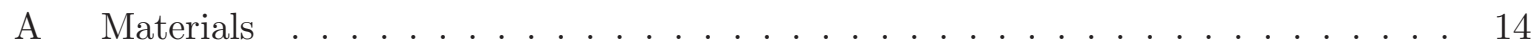

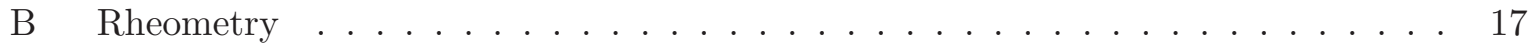

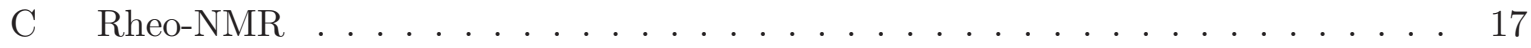

D Rheo-X-ray microtomography . . . . . . . . . . . . . . . . . 19

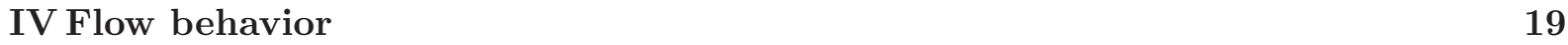

A Main characteristics of the flows . . . . . . . . . . . . . . 19

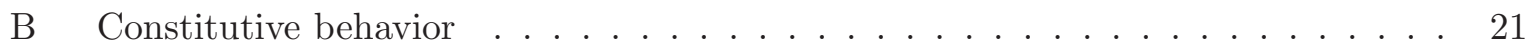

$1 \quad$ Consistency . . . . . . . . . . . . . . . . . . . 22

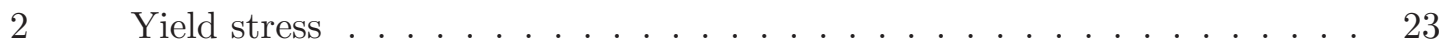

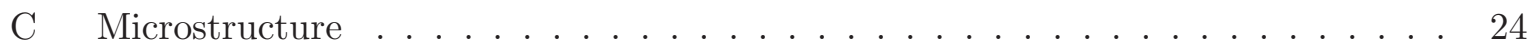

D Static yield stress vs dynamic yield stress: macroscopic measurements . . . . . . 26

E Static yield stress vs dynamic yield stress: velocity profiles . . . . . . . . . . . . 29

F Volume fraction profiles and normal stresses . . . . . . . . . . . . . . . 31

\section{Conclusion}

\section{Introduction}

Dense suspensions involved in industrial processes (concrete casting, drilling muds, foodstuff transport...) and natural phenomena (debris-flows, lava flows...) are usually very polydisperse systems. Many of these systems are yield stress fluids, in which the yield stress primarily arises from the colloidal forces between the smallest suspended particles ${ }^{1}$ (Mewis and Wagner, 2012). When scale separation is possible between these last particles and the largest - noncolloidal - particles in suspension, these materials can be considered in a first step as suspensions of noncolloidal particles embedded in a yield stress fluid [e.g. fresh concrete $\approx$ sand and granulate in cement paste (Geiker et al., 2002; Erdogan, 2005; Toutou and Roussel, 1996; Joumana et al., 2008; Mahaut et al., 2008b); debris-flows $\approx$ rocks in mud (Coussot, 1997; Ancey and Jorrot, 2001)]. Substantial progress in the understanding of the behavior of such materials can thus be made by studying the impact of adding noncolloidal particles to a yield stress fluid of known

\footnotetext{
${ }^{1}$ We do not consider here the case of frictional materials.
} 
properties (Mahaut et al., 2008a; Chateau et al., 2008; Vu et al., 2010). From a more fundamental point of view, these systems, viewed as rigid inclusions in a nonlinear material, may provide crucial tests for micromechanical approaches developed to describe the behavior of composite materials. Model materials with a simple behavior (e.g., close to the ideal elastoplastic behavior) can indeed be designed experimentally (Mahaut et al., 2008a).

Most studies of suspensions of noncolloidal particles have focused on the case of a Newtonian suspending fluid (Stickel and Powell, 2005; Denn and Morris, 2014). Similar to the interstitial fluid, such suspensions have a viscous behavior. Their shear viscosity $\eta(\phi)$ is an increasing function of the particle volume fraction $\phi$ that diverges at the approach of the maximum flowing packing fraction $\phi_{\text {div }}$. This divergence comes from the divergence of the local shear rate and thus of viscous dissipation in the interstices between the particles, which get infinitely small as $\phi$ approaches $\phi_{\text {div }}$ (Frankel and Acrivos, 1967). For an interstitial fluid of viscosity $\eta(0)$, the experimental results are usually well fitted to the phenomenological Krieger-Dougherty law

$$
\eta(\phi)=\eta(0)\left(1-\phi / \phi_{\text {div }}\right)^{-2.5 \phi_{\text {div }}}
$$

consistent with the theoretical value in the dilute limit $\eta(\phi)=\eta(0)(1+2.5 \phi)$. The value of $\phi_{\text {div }}$ depends on the particle shape and on the polydispersity (Vu et al., 2010). Even for monodisperse spheres, there is no consensus on its value (e.g. Boyer et al. (2011b) find $58 \%$ whereas Ovarlez et al. (2006) find $60.5 \%$ ) which may indicate strong dependence on the details of particle interaction at close distance (Sierou and Brady, 2002).

Suspensions in Newtonian fluids also exhibit non-Newtonian behavior, in particular normal stress differences (Zarraga et al., 2000; Boyer et al., 2011a; Couturier et al., 2011; Dbouk et al., 2013; Dai et al., 2013) proportional to the shear rate, which become significant at high shear rates. The $\dot{\gamma}$ dependence of normal stresses originates from hydrodynamic interactions, whereas the emergence of normal stress differences may be related to a shear-induced anisotropy of the microstructure (the particle spatial distribution) (Parsi and Gadala-Maria, 1987; Morris, 2009; Blanc et al., 2013) that is generated by hard sphere repulsions (Brady and Morris, 1997; Blanc et al., 2011a). At a macroscopic scale, these materials are often found to develop volume fraction inhomogeneities (Abbott et al., 1991; Phillips et al., 1992; Tetlow et al., 1998; Shapley et al., 2004; Ovarlez et al., 2006; Altobelli et al., 1991, 1997). E.g., in wide-gap concentric-cylinder Couette flows, particles migrate from the inner to the outer cylinder. Migration is related to shear-induced diffusion (Leighton and Acrivos, 1987a; Acrivos, 1995) in the Leighton and Acrivos (1987b) and Phillips et al. (1992) model: gradients in shear rate generate a particle flux towards the low shear zones (i.e. the outer cylinder in the case of the concentric-cylinder Couette geometry), which is counterbalanced by a particle flux due to viscosity gradients. Steady state is then characterized by an excess of particles in the low shear zones of the flow geometry. There are other models (Nott and Brady, 1994; Mills and Snabre, 1995; Lhuillier, 2009; Nott et al., 2011) in which particle fluxes counterbalance the gradients in particle normal stresses. Although migration is usually slow at low concentration, it can be very rapid and unavoidable at the approach of $\phi_{\text {div }}$ (Ovarlez et al., 2006; Fall et al., 2010, 2015); this poses experimental problems, since the determination of rheological properties with classical (macroscopic) means of investigation requires that the material is homogeneous (Ovarlez et al., 2006).

Noncolloidal particles suspended in yield stress fluids have been the subject of few studies and less is known about their behavior. Moreover, Mahaut et al. (2008a) have shown that the few existing experimental studies do not provide consistent results. Since previous studies have focused on specific materials [generally in link with applications, e.g., particles in a clay dispersion (Coussot, 1997; Ancey and Jorrot, 2001), a cement paste (Geiker et al., 2002), or 
coal slurries (Sengun and Probstein, 1989a,b)], discrepancy between the results may originate from physicochemical interactions between the specific particles and suspending fluid used in each of theses studies. A first problem one has to face when studying these systems is thus the design of model systems that can be described theoretically as rigid particles in a yield stress fluid interacting only through hydrodynamical interactions mediated by the fluid viewed as a continuum. Mahaut et al. (2008a) showed that, provided scale separation is ensured between the particles and the suspending fluid microstructure, and that special care is taken to avoid physicochemical interactions and experimental artefacts, such model materials can indeed be designed and characterized. They have studied the elastoplastic behavior of several suspensions (monodisperse glass or polystyrene beads suspended in concentrated emulsions, Carbopol gels or bentonite suspensions) and found similar rheological behaviors in all systems, thus showing the generic nature of the observed behavior. The results, obtained on model systems, have also been shown to be applicable to more complex systems such as model mortars made of rigid spherical particles in cement pastes (Mahaut et al., 2008b). A major result is that the dimensionless elastic modulus $G^{\prime}(\phi) / G^{\prime}(0)$ and the dimensionless yield stress $\tau_{y}(\phi) / \tau_{y}(0)$ of these systems are related through a simple relationship with no fitting parameter, derived by Chateau et al. (2008) using a micromechanical approach, thus leading to a simple theoretical expression for $\tau_{y}(\phi)$ in agreement with experimental data (more details are given in Sec. II). It thus seems sufficient to characterize only one rheological property of these materials (the elastic modulus) to predict the value of the other one (the yield stress).

The generic viscoplastic properties of such model systems have not been studied experimentally. As for the yield stress, they could be expected to be described by an equation with no fitting parameter once the elastic modulus is known (Chateau et al., 2008), provided the microstructure is not significantly changed by the flow. However, the spatial distribution of particles is known to depend on flow history in suspensions (an anisotropic distribution is induced by shear), which has an impact on their properties (Morris, 2009; Blanc et al., 2011b, 2013). Since the Chateau et al. (2008) and Mahaut et al. (2008a) works have dealt with isotropic suspensions only, there is thus first a need to extend the theoretical approach to the case of sheared (thus, likely anisotropic) suspensions, and to characterize the material shear-dependent microstructure. We emphasize that, by contrast with a Newtonian interstitial fluid, the particle distribution in space can now be expected to depend on the shear rate due to the nonlinear behavior of the interstitial fluid.

When dealing with the flow properties of a suspension of noncolloidal particles, an experimental difficulty may occur: shear-induced migration. As far as we know, migration in yield stress fluids has not been studied in the literature and remains to be investigated. In rheological studies, it is usually considered as an artefact that has to be avoided when possible. As it may not always be avoided, in particular at high particle volume fractions, one has to find a way to take it into account; a possible way consists in measuring the local velocity and volume fraction fields (e.g., with rheo-NMR methods [Callaghan (1999)]) and in using this information to infer the rheological properties values as a function of the locally measured volume fraction (Ovarlez et al., 2006).

In this paper, we investigate the flow behavior of suspensions of particles in yield stress fluids. Following Mahaut et al. (2008a), we use polystyrene beads in a concentrated emulsion as a model system. Flows are studied with the use of a rheometer inserted in a magnetic resonance imager which allows the measurement of velocity and volume fraction fields, and accounting for shearinduced migration when determining the rheological behavior of the suspensions. We also use a rheometer coupled to a X-ray microtomograph to characterize the suspensions' microstructure.

In Sec. II, we discuss the theoretical behavior of suspensions of rigid particles in linear and 
nonlinear media; we extend the theoretical approach of Chateau et al. (2008) to describe suspensions in simple shear flows, which are anisotropic. In Sec. III, we present the model system used in this work, the experimental setups and the methods. The experimental results are presented in Sec. IV: we study the evolution of the flow properties with the particle volume fraction, and we discuss the emergence of flow inhomogeneities and of a shear-dependent microstructure; throughout this part, we compare the experimental results and the theoretical predictions.

\section{Theory}

In this section, we follow the same steps as Chateau et al. (2008) to obtain theoretical estimates for the viscoplastic properties of suspensions of spherical particles in yield stress fluids. The estimates derived in this previous work are theoretically valid for isotropic suspensions only. They were validated on such model materials (Mahaut et al., 2008a), which was possible only for properties at rest (elastic modulus) and at flow start (static yield stress). To deal with steady-state flow properties, we must investigate the case of anisotropic materials, since sheared suspensions are known to develop an anisotropic microstructure (anisotropic particle spatial distribution [Morris (2009); Blanc et al. (2013)]), the signature of which are normal stress differences in simple shear flows (Zarraga et al., 2000; Morris, 2009). In the following, we show that the same approach can be conducted as in Chateau et al. (2008) for an anisotropic suspension, but only in the case of a simple shear flow, and for an isotropic interstitial material. This allows us in particular to derive expressions for normal stress differences and to propose predictions for the steady state volume fraction profiles in a concentric-cylinder Couette geometry.

In the following, the stress tensor is denoted $\sigma_{i j}$, the deviatoric stress tensor is denoted $\tau_{i j}$, the strain tensor is denoted $\varepsilon_{i j}$, and the strain rate tensor is denoted $d_{i j}$. The shear stress is $\tau=\sqrt{\frac{1}{2} \tau_{i j} \tau_{i j}}$, the shear strain is $\gamma=\sqrt{2 \varepsilon_{i j} \varepsilon_{i j}}$ and the shear rate is $\dot{\gamma}=\sqrt{2 d_{i j} d_{i j}}$. In the case of a simple shear flow, the flow is described only by $\gamma$ and $\dot{\gamma}$, the stress is described by $\tau$ and by two normal stress differences $N_{1}=\sigma_{11}-\sigma_{22}=\tau_{11}-\tau_{22}$ and $N_{2}=\sigma_{22}-\sigma_{33}=\tau_{22}-\tau_{33}$; indexes 1,2 , and 3 , correspond, respectively, to the velocity direction, to the velocity gradient direction, and to the vorticity direction.

We consider monodisperse ${ }^{2}$ rigid particles of diameter $d$ dispersed in a fluid at a volume fraction $\phi$. Our approach describes the general case of a nonlinear suspending fluid (which includes yield stress fluids). As will be discussed in the following, the theoretical approach works strictly for an isotropic suspending fluid only. We thus assume that it can be described by a generalized Newtonian behavior

$$
\tau_{i j}=2 \eta(\dot{\gamma}) d_{i j}
$$

i.e., $\tau=\eta(\dot{\gamma}) \dot{\gamma}$ in a simple shear flow, where $\eta(\dot{\gamma})$ is the shear-rate-dependent apparent viscosity of the material. In the case of a suspending yield stress fluid, we will consider that the material flow behavior is described by the Herschel-Bulkley behavior for applied shear stresses $\tau$ above a yield stress $\tau_{y}(0)$ (which correspond to a von Mises criterion):

$$
\tau_{i j}=2 \frac{\tau_{y}(0)+\eta_{H B}(0) \dot{\gamma}^{n(0)}}{\dot{\gamma}} d_{i j} \text { when } \tau \geq \tau_{y}(0)
$$

where $\eta_{H B}(0)$ is the consistency, and $n(0)$ the index; in simple shear, this yields:

$$
\tau=\tau_{y}(0)+\eta_{H B}(0) \dot{\gamma}^{n(0)} \text { when } \tau \geq \tau_{y}(0)
$$

\footnotetext{
${ }^{2}$ Some aspects of the linear and nonlinear behavior of polydisperse suspensions are discussed in Vu et al. (2010).
} 
This corresponds to the behavior described by Eq. 2 with $\eta(\dot{\gamma})=\left(\tau_{y}(0)+\eta_{H B}(0) \dot{\gamma}^{n(0)}\right) / \dot{\gamma}$.

\section{A Homogenization approach}

In order to predict the behavior of a composite material such as a suspension, one usually computes the stresses at the particle scale, which requires the evaluation of the local strain rate field $d_{i j}^{\text {local }}(\vec{x})$ in the fluid around the particles when a homogeneous strain rate $d_{i j}$ is applied to the suspension; here $\vec{x}$ denote the 3D position vector in the suspension. Instead of trying to infer all properties from the (difficult) computation of $d_{i j}^{\text {local }}(\vec{x})$, the method used by Chateau et al. (2008) consists, first, in assuming that the overall properties of the suspension can be accurately estimated from an average estimate $\overline{\dot{\gamma}}_{\text {local }}$ of the local shear rate $\dot{\gamma}_{\text {local }}(\vec{x})$ over the suspending fluid domain, second, in using one of the material properties determined experimentally to estimate the value of $\overline{\dot{\gamma}}_{\text {local }}$, and finally, in using this last value to predict the other properties. These various steps are described more precisely in the subsections below.

\section{Choice of a local strain rate estimate}

It is shown in Chateau et al. (2008) that accurate estimates of the overall properties of the suspension are obtained by using

$$
\overline{\dot{\gamma}}_{\text {local }}(\phi)=\sqrt{<\dot{\gamma}_{\text {local }}^{2}(\vec{x})>}
$$

as the local strain rate estimate in the interstitial material. Here, $\langle A\rangle$ denotes the average of $A$ over the suspending fluid domain. This nontrivial choice is not arbitrary and is an essential step in the derivation of the suspension behavior. Indeed, as discussed in Chateau et al. (2008), this choice has been shown to be optimal in the framework of a variational approach to the solution of the nonlinear homogenization problem under consideration. A simpler and a priori more natural choice would have been to use $\overline{\dot{\gamma}}_{\text {local }}(\phi)=<\dot{\gamma}_{\text {local }}(\vec{x})>=\dot{\gamma} /(1-\phi)$, but Suquet (1997) has shown that estimates of the overall properties of the heterogeneous material obtained using this effective liquid strain rate are less accurate than those obtained using Eq. 5. It should be noted that the fact that Eq. 5 is an optimal choice has been shown theoretically for isotropic composite materials; in the absence of such a result for anisotropic materials, we will consider in the following that this remains the best possible estimate of the local strain rate in the interstitial fluid in anisotropic suspensions.

\section{Computation of the local strain rate estimate}

The second step consists in assuming that, for a given microstructure for the suspension, the solution of the linear problem is known, and to use this information to compute $\overline{\dot{\gamma}}_{\text {local }}(\phi)$. The linear problem is the estimation of the overall properties of a dispersion of particles in a Hookean material, or, equivalently, of a suspension of particles in a Newtonian material.

For a suspension of particles in a Newtonian fluid of viscosity $\eta(0)$ in a simple shear flow of shear rate $\dot{\gamma}$, the suspension is a priori characterized by stress components that vary linearly with $\dot{\gamma}$ :

$$
\begin{aligned}
\tau & =g(\phi) \eta(0) \dot{\gamma} \\
N_{1} & =h_{1}(\phi) \eta(0) \dot{\gamma}=\frac{h_{1}(\phi)}{g(\phi)} \tau \\
N_{2} & =h_{2}(\phi) \eta(0) \dot{\gamma}=\frac{h_{2}(\phi)}{g(\phi)} \tau=\frac{h_{2}(\phi)}{h_{1}(\phi)} N_{1}
\end{aligned}
$$


The functions $g(\phi), h_{1}(\phi)$, and $h_{2}(\phi)$, depend on the microstructure. They are known for isotropic suspensions: in this case, $h_{1}(\phi)=h_{2}(\phi)=0$, and $g(\phi)$ is the so-called dimensionless high frequency dynamic viscosity (Sierou and Brady, 2002); $g(\phi)$ for isotropic suspensions has also been characterized experimentally by Mahaut et al. (2008a). For a steadily sheared suspension, data for $g(\phi), h_{1}(\phi)$, and $h_{2}(\phi)$ can also be found in the literature (Boyer et al., 2011b; Dbouk et al., 2013; Dai et al., 2013; Denn and Morris, 2014); in this last case, the (anisotropic) suspension microstructure is fixed by shear.

One can a priori imagine any microstructure for the suspension: the 'instantaneous' behavior of the suspension would then be characterized by different functions $g(\phi), h_{1}(\phi)$, and $h_{2}(\phi)$, which could be measured by applying infinitesimal nonperturbative oscillations to the material. The multiplicity of possible $g(\phi), h_{1}(\phi)$, and $h_{2}(\phi)$ values is seen, e.g., in shear reversal experiments where suspensions see their microstructure changing continuously (Blanc et al., 2013) together with their macroscopic properties (Narumi et al., 2002; Blanc et al., 2011b). At this stage, we will make no hypothesis on their value for the considered suspensions; we simply note that $g(\phi), h_{1}(\phi)$, and $h_{2}(\phi)$ bear some information on the suspension microstructure that can be used to predict the behavior of suspensions of particles in non-Newtonian fluids with the same microstructure.

With this knowledge, the local strain rate estimate $\overline{\dot{\gamma}}_{\text {local }}(\phi)$ in a suspension of given microstructure can be computed in some cases by matching the density of energy $e$ dissipated at the macroscopic scale in the suspension in a Newtonian fluid, and that dissipated at the local scale in the Newtonian interstitial fluid, $e_{\text {local }}$. It is thus assumed here that there is negligible dissipation from particle direct contacts (sliding friction or collisions). It seems to be a reasonable assumption for particle volume fractions $\phi \lesssim 0.4$; at larger volume fraction, one has to be cautious since, as shown by Gallier et al. (2014), contact forces provide the main contribution to the suspension shear stress; with a nonzero interparticle coefficient of friction, there is thus likely non-negligible frictional dissipation. In dense suspensions, close to jamming, the presented approach can thus probably not be conducted as is.

The density of energy dissipated at the local scale in the interstitial fluid, $e_{\text {local }}$, is

$$
\begin{aligned}
e_{\text {local }} & =(1-\phi)<\tau_{i j}^{\text {local }}(\vec{x}) d_{i j}^{\text {local }}(\vec{x})>=(1-\phi)<\eta(0) \dot{\gamma}_{\text {local }}^{2}(\vec{x})> \\
& =(1-\phi) \eta(0) \overline{\dot{\gamma}}_{\text {local }}^{2}(\phi)
\end{aligned}
$$

This quantity is here computed easily due to the fact that the interstitial material has an isotropic behavior (Newtonian), which is thus an important assumption. For a linear anisotropic interstitial fluid, due to the complexity of the strain rate field in the interstitial fluid, $\tau_{i j}^{\text {local }} d_{i j}^{\text {local }}$ would not be simply proportional to $\dot{\gamma}_{\text {local }}^{2}$ but would be also a function of another invariant of the strain rate tensor.

The density of energy $e$ dissipated at the macroscopic scale can also be computed as a function of $\dot{\gamma}$ only, in two situations: (i) for an isotropic suspension, in which case $\tau_{i j} d_{i j}=$ $2 \eta(0) g(\phi) d_{i j} d_{i j}=\eta(0) g(\phi) \dot{\gamma}^{2}$ for any prescribed strain rate $d_{i j}$; (ii) for an anisotropic suspension in a simple shear flow only, in which case normal stress differences do not produce any mechanical work, and $\tau_{i j} d_{i j}=\eta(0) g(\phi) \dot{\gamma}^{2}$ is independent of $h_{1}(\phi)$ and $h_{2}(\phi)$. In both cases, we finally get

$$
e=\eta(0) g(\phi) \dot{\gamma}^{2}
$$

By matching the density of energy dissipated at the macroscopic scale, and its microscopic origin, that is, the density of energy dissipated in the interstitial fluid, we finally obtain a simple relationship between the macroscopic shear rate $\dot{\gamma}$ and the estimate of the local strain rate:

$$
\overline{\dot{\gamma}}_{\text {local }}(\phi)=\dot{\gamma} \sqrt{g(\phi) /(1-\phi)}
$$


All the useful information on the material microstructure and on the way the material is sheared locally is thus supposed to be encoded in $g(\phi)$.

\section{From linear to nonlinear properties}

The next step consists in using the information obtained on $\overline{\dot{\gamma}}_{\text {local }}$ to predict the value of the nonlinear properties. Chateau et al. (2008) have linearized the suspending fluid behavior (Eq. 2) at each prescribed macroscopic shear rate $\dot{\gamma}$, and have used Eq. 5 to estimate its apparent viscosity which is thus $\eta\left(\overline{\dot{\gamma}}_{\text {local }}\right)$. Using Eq. 12, the suspending fluid apparent viscosity is then estimated as $\eta(\dot{\gamma} \sqrt{g(\phi) /(1-\phi)})$, which is used to predict the behavior of the linearized suspension behavior associated to a shear rate $\dot{\gamma}$.

Two situations shall then be distinguished, as above. For an isotropic suspension, the macroscopic apparent viscosity is simply $g(\phi)$ times the suspending fluid apparent viscosity, and the full tensorial behavior of the suspension can be estimated as:

$$
\tau_{i j}=2 \eta(\dot{\gamma} \sqrt{g(\phi) /(1-\phi)}) g(\phi) d_{i j}
$$

For an anisotropic suspension, the behavior in simple shear can be estimated as

$$
\begin{aligned}
\tau & =g(\phi) \eta(\dot{\gamma} \sqrt{g(\phi) /(1-\phi)}) \dot{\gamma} \\
N_{1} & =h_{1}(\phi) \eta(\dot{\gamma} \sqrt{g(\phi) /(1-\phi)}) \dot{\gamma} \\
N_{2} & =h_{2}(\phi) \eta(\dot{\gamma} \sqrt{g(\phi) /(1-\phi)}) \dot{\gamma}
\end{aligned}
$$

Within this approach, the ratios of shear to normal stresses remain naturally unchanged when changing the suspending fluid, which comes from their independence on the interstitial fluid viscosity, i.e.:

$$
N_{1}=\frac{h_{1}(\phi)}{g(\phi)} \tau \quad \text { and } \quad N_{2}=\frac{h_{2}(\phi)}{g(\phi)} \tau=\frac{h_{2}(\phi)}{h_{1}(\phi)} N_{1}
$$

This will be shown to have important consequences when modeling migration in Sec. II C.

An important assumption that has been made above is that the same functions $g(\phi), h_{1}(\phi)$ and $h_{2}(\phi)$ can be used to estimate the linearized suspension characteristics independently of $\dot{\gamma}$. In other words, it is assumed that the microstructure does not vary significantly with $\dot{\gamma}$. Although it has no reason to be true for nonlinear suspending fluids, this has been somehow validated for particles in power-law fluids by Liard et al. (2014), who have shown that distributions of shear rates in the interstitial fluid are similar for power-law and Newtonian interstitial fluids. One has to keep in mind, however, that this might not be true for other complex fluids, as will be shown below for the yield stress fluids we study (see Sec. IV C); changes in the microstructure as a function of the shear rate specific to nonlinear interstitial fluids might then have a significant impact on the value of the rheological properties. Nevertheless, even in such cases, it is possible to use the above estimates in ranges of shear rates characterized by a similar microstructure; different $g(\phi), h_{1}(\phi)$, and $h_{2}(\phi)$ functions shall then be used in these different ranges.

\section{Application to yield stress fluids}

In the case of a suspending fluid described by a Herschel-Bulkley behavior (Eq. 3), for both isotropic and anisotropic suspensions, the shear stress $\tau$ in the suspension is found to be:

$$
\begin{aligned}
\tau & =\sqrt{(1-\phi) g(\phi)}\left[\tau_{y}(0)+\eta_{H B}(0)\left(\frac{g(\phi)}{1-\phi}\right)^{n(0) / 2} \dot{\gamma}^{n(0)}\right] \\
& =\tau_{y}(\phi)+\eta_{H B}(\phi) \dot{\gamma}^{n(\phi)}
\end{aligned}
$$


It is then first seen that

$$
n(\phi)=n(0)
$$

i.e., the suspension shear stress is that of a Herschel-Bulkley material of same index $n \equiv n(0)$ as the interstitial fluid ${ }^{3}$. The dimensionless yield stress is

$$
\tau_{y}(\phi) / \tau_{y}(0)=\sqrt{(1-\phi) g(\phi)}
$$

and the dimensionless consistency is

$$
\eta_{H B}(\phi) / \eta_{H B}(0)=\sqrt{(g(\phi))^{n+1}(1-\phi)^{1-n}}
$$

which can also be rewritten as

$$
\eta_{H B}(\phi) / \eta_{H B}(0)=\left(\tau_{y}(\phi) / \tau_{y}(0)\right)^{n+1}(1-\phi)^{-n}
$$

It is worth noting that, within this approach, the knowledge of a single mechanical property is sufficient to help predicting the value of all other properties. Eq. 23 shows in particular that the validity of the homogenization approach can be tested without the knowledge of the linear properties of the material.

For an isotropic suspension, the material tensorial behavior is an isotropic Herschel-Bulkley behavior (Eq. 3) of index $n$, and yield stress and consistency given by Eqs. 21 and 22. For an anisotropic suspension in simple shear, the shear stress is given by Eq. 18, and the material is also predicted to be characterized by normal stress differences

$$
\begin{aligned}
N_{1} & =h_{1}(\phi) \frac{\tau_{y}(0)+\eta_{H B}(0) \dot{\hat{\gamma}}_{\text {local }}(\phi)^{n}}{\dot{\bar{\gamma}}_{\text {local }}(\phi)} \dot{\gamma} \\
& =\frac{h_{1}(\phi)}{\sqrt{g(\phi) /(1-\phi)}}\left[\tau_{y}(0)+\eta_{H B}(0)(g(\phi) /(1-\phi))^{n / 2} \dot{\gamma}^{n}\right]
\end{aligned}
$$

and

$$
N_{2}=\frac{h_{2}(\phi)}{\sqrt{g(\phi) /(1-\phi)}}\left[\tau_{y}(0)+\eta_{H B}(0)(g(\phi) /(1-\phi))^{n / 2} \dot{\gamma}^{n}\right]
$$

It is predicted in particular that these suspensions exhibit 'yield normal stress differences'.

\section{Domain of validity}

It is worth reminding the main assumptions made in all the above derivations, in order to better understand the domain of validity of the developed equations:

- Eq. 5 has been chosen as a local strain rate estimate. It has been shown to be an optimal choice for isotropic suspensions only. The same results remains to be shown rigorously for anisotropic suspensions.

- The interstitial fluid has been assumed to have an isotropic behavior. This assumption is necessary to compute the suspension stresses by using Eqs. 6, 7, and 8 when linearizing the fluid and suspension behaviors. It is also used when calculating the density of energy dissipated in the complex flow at the local scale (Eqs. 9 and 10) to compute the local strain rate estimate. In the absence of baseline results for suspensions of particles in linear anisotropic fluids, the case of a nonlinear anisotropic interstitial fluid cannot yet be treated with the same approach.

\footnotetext{
${ }^{3}$ For simplicity, we will use $n$ to denote the index of both the suspending fluid and the suspension in the sequel.
} 
- In computing the energy dissipated at the local scale, it was also assumed that there is negligible dissipation from particle direct contacts (sliding friction or collision). A consequence is that the presented approach may not work at high volume fraction. A finer micromechanical description of the suspensions would probably be necessary to describe cases where interparticle dissipation is not negligible.

- The case of anisotropic suspensions has been handled here only in the case of simple shear flows. In this case, normal stresses do not produce any mechanical work, which makes it possible to derive equations depending on a single function $(g(\phi))$. It would a priori be possible to develop the same approach for other flows, provided the behavior of suspensions in Newtonian fluids is well known for these flows; this would, however, probably yield more complex and less tractable expressions.

- It should also be noted that our prediction for $\tau_{y}(\phi)$ concerns the limit shear stress of the material in simple shear, and not its 'true' yield stress (as would be defined from a yield criterion based on the full stress tensor). Indeed, the yield criterion of the suspension should probably take into account its normal stress differences (which are different from zero at $\dot{\gamma}=0$ ); this might have important consequences on the flow properties of the material at the solid/liquid transition (Cheddadi et al., 2012). Building a 3D yield criterion for yield stress suspensions remains a challenging question.

- $g(\phi)$ has been assumed to be uniquely defined for anisotropic suspensions, independently of the fluid and of $\dot{\gamma}$, which seems to be a good approximation for power-law fluids (Liard et al., 2014). This is useful to get simple equations such as Eq. 23 and to derive all quantities from the sole knowledge of $g(\phi)$; this is useful also because $g(\phi)$ is well characterized for sheared viscous suspensions, which allows deriving phenomenological expressions for nonlinear interstitial fluids (see Sec. II B). $\dot{\gamma}$-dependent $g(\phi)$ can nevertheless be handled in Eqs. 14, 15, and 16. Their determination remains however challenging.

\section{B Phenomenological expressions}

For both isotropic and anisotropic suspensions, the development of phenomenological expressions for the yield stress and the consistency relies on the existence of phenomenological expressions for the linear response $g(\phi)$. The yield stress fluids we study have a linear elastic behavior below their yield stress: $\tau_{i j}=G^{\prime}(0) \gamma_{i j}$ when $\tau<\tau_{y}$. The best way to get $g(\phi)$ thus seems to determine it directly on the materials we study as $g(\phi)=G^{\prime}(\phi) / G^{\prime}(0), G^{\prime}(\phi)$ being the elastic modulus of the suspension at particle volume fraction $\phi . g(\phi)$ can also a priori be obtained from the response of any suspension of particles in a linear (Hookean or Newtonian) material with the same structure as the studied yield stress suspension.

From now, we will distinguish $g_{\text {iso }}(\phi)$ and $g_{\text {aniso }}(\phi)$ for, respectively, isotropic and anisotropic suspensions.

\section{Isotropic suspensions}

Mahaut et al. (2008a) have measured both the elastic modulus (Fig. 1a) and the yield stress (Fig. 1b) of isotropic suspensions of particles in yield stress fluids; measuring their consistency is not possible as the isotropic structure is destroyed by flow. The dimensionless elastic modulus of the suspensions is found to be well fitted to the Krieger-Dougherty equation up to $\phi=50 \%$ (see Fig. 1a), as classically found for the dimensionless viscosity of suspensions in Newtonian 
(a)

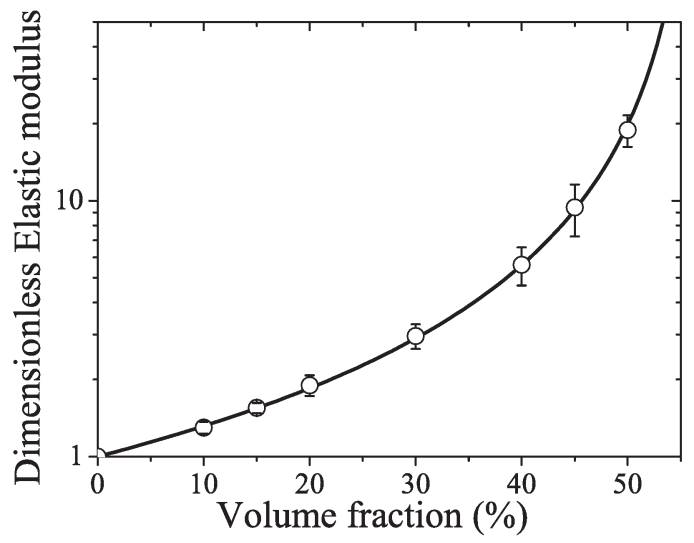

(b)

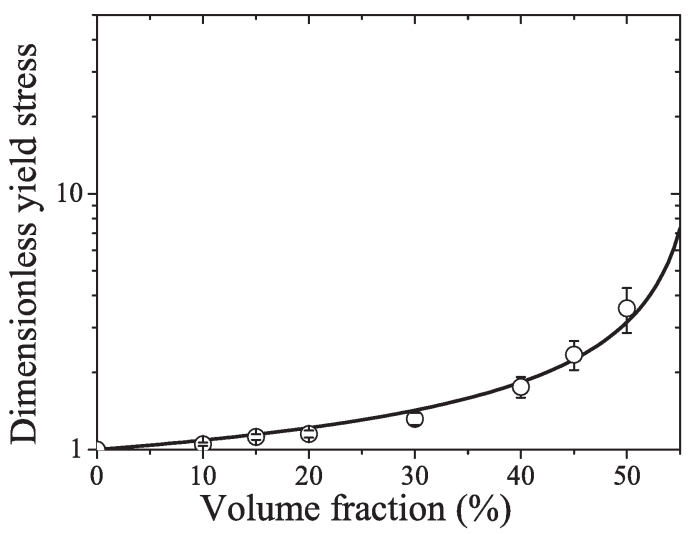

Figure 1: Dimensionless elastic modulus $G^{\prime}(\phi) / G^{\prime}(0)$ (a) and dimensionless static yield stress $\tau_{y}(\phi) / \tau_{y}(0)$ (b) measured in isotropic suspensions of monodisperse spherical particles in yield stress fluids by Mahaut et al. (2008a) (data are averages over 13 different suspensions). The line in Fig. 1a is a Krieger-Dougherty equation (Eq. 27), the line in Fig. 1b is the equation derived by Chateau et al. (2008) (Eq. 28).

fluids [this is due to the similarity of the elastic and viscous problems (Torquato, 2002)]:

$$
g_{\text {iso }}(\phi)=G^{\prime}(\phi) / G^{\prime}(0)=\left(1-\phi / \phi_{\text {div }}\right)^{-2.5 \phi_{\text {div }}} \quad \text { with } \quad \phi_{\text {div }}=0.57
$$

Combining Eqs. 21 and 27 then yields a simple phenomenological law for the yield stress:

$$
\tau_{y}(\phi) / \tau_{y}(0)=\sqrt{(1-\phi)\left(1-\phi / \phi_{\mathrm{div}}\right)^{-2.5 \phi_{\mathrm{div}}}} \quad \text { with } \quad \phi_{\mathrm{div}}=0.57
$$

This expression is in very good agreement with the observations of Mahaut et al. $(2008 \mathrm{a}, \mathrm{b})$ for the static yield stress of isotropic yield stress suspensions, for $\phi \leq 50 \%$ (Fig. 1b). This validates Eqs. 12 and 21, and shows that the yield stress of these suspensions can be derived from the sole knowledge of their elastic modulus.

\section{Anisotropic suspensions}

In principle, we could use the same approach as above to determine directly $g_{\text {aniso }}(\phi)$ on the studied suspensions from the measurement of the suspension elastic modulus after flow cessation or by using parallel superposition (Vermant et al., 1998). This cannot be done easily, however, at volume fractions $\phi \gtrsim 40 \%$, due to volume fraction inhomogeneities (see Sec. IV A); parallel superposition poses other problems in yield stress fluids, such as accounting for possible flow inhomogeneities.

Another possibility consists in studying the same particles in a Newtonian fluid of viscosity $\eta(0)$ and to assume that the suspension microstructure is the same as in the yield stress fluid in order to get $g_{\text {aniso }}(\phi)$ as $g_{\text {aniso }}(\phi)=\eta(\phi) / \eta(0)$. This is the approach chosen by Liard et al. (2014) and Dagois-Bohy et al. (2015). This approach poses several problems. First, we will show in Sec. IV C that the suspension microstructure depends on the shear rate in a yield stress fluid, which contrasts with the case of a Newtonian interstitial fluid. Second, for given particles, the function $g_{\text {aniso }}(\phi)$ depends on the (Newtonian) interstitial fluid that is chosen, as can be 


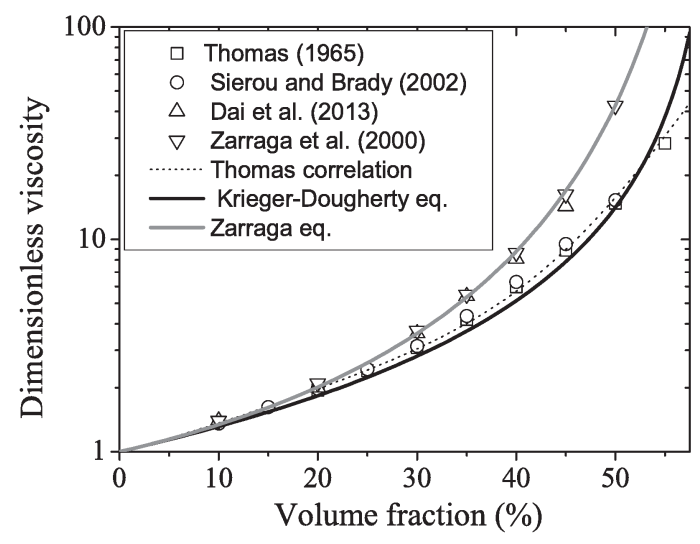

Figure 2: Dimensionless viscosity $g_{\text {aniso }}(\phi)=\eta(\phi) / \eta(0)$ obtained in steady-state simple shear flows of monodisperse spherical particles in Newtonian fluids: data replotted from the literature (see legend). The black line is Eq. 29; the dotted line is a correlation proposed by Thomas (1965). The grey line is Eq. 30.

seen from a survey of the literature. E.g., the same PS particles (same size: $40 \mu \mathrm{m}$, same provider: Dynoseeds) lead to $\left(1-\phi / \phi_{\text {div }}\right)^{-2}$ scaling for the viscosity divergence in a silicone oil (Bonnoit et al., 2010) whereas Fall et al. (2010) report a $\left(1-\phi / \phi_{\text {div }}\right)^{-1}$ scaling in water. In addition, for similar PS beads as those that we use (same provider: Dynoseeds), the viscosity was observed to diverge at $\phi_{\text {div }}=60.5 \%$ for particles dispersed in a silicone oil (Ovarlez et al., 2006) and in water (Fall et al., 2010), whereas $\phi_{\text {div }}=58 \%$ was obtained by Boyer et al. (2011b) in polyethylene glycol. These differences likely come from the strong dependence of the suspension behavior on the short range interactions between the particles (Brady and Morris, 1997; Sierou and Brady, 2002), which affects the cut-off on lubrication forces, and on the interparticle friction coefficient (Sierou and Brady, 2002; Gallier et al., 2014). Friction and interparticle interactions most probably depend on the physicochemical properties of the interstitial fluid.

We emphasize that this implies that one cannot rely on measurements performed with beads in a given Newtonian fluid to predict quantitatively the behavior of the same beads in another material. Instead of trying to study the range of possible viscosities for our beads in Newtonian fluids by using many different fluids, we have thus chosen to rely on results of the literature.

To describe the possible linear response of sheared viscous suspensions, we thus use the sets of data shown in Fig. 2, which seem to provide lower and upper limits for values observed in the literature; e.g., at $\phi=50 \%$, the data of Zarraga et al. (2000) and those of Thomas (1965) differ by a factor 3 . The data reported by Thomas (1965) come from his analysis of many works of the literature; they are consistent with the simulations of Sierou and Brady (2002), and may thus correspond to cases where interparticle friction does not contribute much to dissipation. The correlation proposed by Thomas (1965), plotted in Fig. 2, does not diverge at high $\phi$. We thus chose to describe these data with the Krieger-Dougherty equation (Eq. 29), with $\phi_{\text {div }}=0.605$ consistent with the finding of Ovarlez et al. (2006), in fair agreement with the Thomas (1965) and Sierou and Brady (2002) data. Zarraga et al. (2000) have proposed another equation (Eq. 30) which provides a good fit to their data and to those of Dai et al. (2013).

In the following, we will thus test two expressions for $g_{\text {aniso }}$ :

$$
g_{\text {aniso }, 1}(\phi)=\left(1-\phi / \phi_{\text {div }}\right)^{-2.5 \phi_{\text {div }}} \quad \text { with } \quad \phi_{\text {div }}=0.605
$$


and

$$
g_{\text {aniso }, 2}(\phi)=\left(1-\phi / \phi_{\text {div }}\right)^{-3} e^{-2.34 \phi} \quad \text { with } \quad \phi_{\text {div }}=0.62
$$

These expressions yield, respectively

$$
\begin{array}{rlll}
\tau_{y}(\phi) / \tau_{y}(0)=\sqrt{(1-\phi)\left(1-\phi / \phi_{\text {div }}\right)^{-2.5 \phi_{\text {div }}}} & \text { with } & \phi_{\text {div }}=0.605 \\
\eta_{H B}(\phi) / \eta_{H B}(0)=\sqrt{\left(1-\phi / \phi_{\text {div }}\right)^{-2.5(n+1) \phi_{\text {div }}}(1-\phi)^{1-n}} & \text { with } & \phi_{\text {div }}=0.605
\end{array}
$$

and

$$
\begin{array}{rll}
\tau_{y}(\phi) / \tau_{y}(0)=\sqrt{(1-\phi)\left(1-\phi / \phi_{\text {div }}\right)^{-3} e^{-2.34 \phi}} & \text { with } & \phi_{\text {div }}=0.62 \\
\eta_{H B}(\phi) / \eta_{H B}(0)=\sqrt{\left(1-\phi / \phi_{\text {div }}\right)^{-3(n+1)} e^{-2.34(n+1) \phi}(1-\phi)^{1-n}} & \text { with } & \phi_{\text {div }}=0.62
\end{array}
$$

\section{Normal stress differences and shear-induced migration}

Normal stress differences are particularly important when dealing with shear-induced migration. Shear-induced migration is indeed driven by a gradient in particle normal stresses (Nott and Brady, 1994; Mills and Snabre, 1995; Morris and Boulay, 1999; Lhuillier, 2009; Nott et al., 2011). Noting $\Sigma_{i j}^{p}$ the particle stresses, the particle normal stresses are

$$
\Sigma_{11}^{p}=\alpha_{1}(\phi) \eta(0) \dot{\gamma}, \quad \Sigma_{22}^{p}=\alpha_{2}(\phi) \eta(0) \dot{\gamma}, \quad \Sigma_{33}^{p}=\alpha_{3}(\phi) \eta(0) \dot{\gamma}
$$

in a Newtonian fluid of viscosity $\eta(0)$, where $\alpha_{i}(\phi)$ are the dimensionless particle normal stress. Recent experimental values for particle normal stresses can be found in Boyer et al. (2011b); Dbouk et al. (2013); Garland et al. (2013). We recall that indexes 1, 2, and 3, correspond, respectively, to the velocity direction, to the velocity gradient direction, and to the vorticity direction. This can be rewritten as:

$$
\Sigma_{11}^{p}=\frac{\alpha_{1}(\phi)}{g(\phi)} \tau, \quad \Sigma_{22}^{p}=\frac{\alpha_{2}(\phi)}{g(\phi)} \tau, \quad \Sigma_{33}^{p}=\frac{\alpha_{3}(\phi)}{g(\phi)} \tau
$$

In the linearized yield stress fluid, Eq. 36 should remain valid with $\tau$ given by Eq. 18 .

Fully modeling shear-induced migration and its kinetics is far out of the scope of the present paper. Nevertheless, as we are interested here in steady flow properties of suspensions of particles in yield stress fluids, we need to understand the steady volume fraction profiles generated by migration in these suspensions.

Steady-state volume fraction profiles can easily be predicted by ensuring stress balance at both the suspension and the particle phase scale: $\phi(\vec{r})$ profiles are found from solving $\partial_{i} \sigma_{i j}=0$ and $\partial_{i} \Sigma_{i j}^{p}=0$, with $\phi(\vec{r})$ a free function (Morris and Boulay, 1999; Lhuillier, 2009; Nott et al., 2011).

For the concentric-cylinder Couette flow studied here, the relevant equations are (Morris and Boulay, 1999)

$$
\begin{aligned}
& \partial_{r}\left(r^{2} \tau\right)=0 \\
& \partial_{r} \Sigma_{r r}^{p}=\frac{\Sigma_{\theta \theta}^{p}-\Sigma_{r r}^{p}}{r}
\end{aligned}
$$

Thanks to Eq. 36, Eq. 38 can be changed into

$$
\tau \partial_{r}\left[\frac{\alpha_{2}(\phi(r))}{g(\phi(r))}\right]+\left[\frac{\alpha_{2}(\phi(r))}{g(\phi(r))}\right] \partial_{r} \tau=\frac{\alpha_{1}(\phi(r))-\alpha_{2}(\phi(r))}{g(\phi(r)) r} \tau
$$




\begin{tabular}{|c|c|c|}
\hline & Yield stress fluids: & Particles: \\
& Concentrated emulsions & Spherical beads \\
\hline Rheo-NMR & $\mathrm{CaCl}_{2}$ water solution $(72 \%)$ in dodecane \\
and & droplet size: $1 \mu \mathrm{m}$; dispersity: $20 \%$ & PS particles \\
macroscopic & behavior: $\tau=\tau_{y}+\eta_{H B} \dot{\gamma}^{0.5}$ & diameter: $250 \mu \mathrm{m}$; dispersity: $5 \%$ \\
measurements & with $\tau_{y}=22 \mathrm{~Pa}, \eta_{H B}=5.3 \mathrm{~Pa} . \mathrm{s}^{0.5}$ & \\
\hline & NaI water solution $(77.5 \%)$ in dodecane & PS particles \\
Rheo-X-ray & droplet size: $1 \mu \mathrm{m} ;$ dispersity: $20 \%$ & diameter: $140 \mu \mathrm{m}$; dispersity: $5 \%$ \\
tomography & behavior: $\tau=\tau_{y}+\eta_{H B} \dot{\gamma}^{0.5}$ & vol. fraction: $37 \%$ \\
& with $\tau_{y}=26 \mathrm{~Pa}, \eta_{H B}=5.1$ Pa.s & \\
\hline
\end{tabular}

Table 1: Model suspensions of particles in yield stress fluid used in the experiments.

When combined with Eq. 37 this finally yields

$$
\partial_{r}\left[\frac{\alpha_{2}(\phi(r))}{g(\phi(r))}\right]=\frac{\alpha_{1}(\phi(r))+\alpha_{2}(\phi(r))}{g(\phi(r)) r}
$$

which, once the material properties $\alpha_{1}(\phi), \alpha_{2}(\phi)$ and $g(\phi)$ are known, allows one to determine the steady-state inhomogeneous volume fraction profile $\phi(r)$.

It is striking here is that it is the exact same equation as for particles suspended in a Newtonian medium (Morris and Boulay, 1999). In other words, it is predicted that steady volume fraction profiles in a concentric-cylinder Couette geometry should be the same in yield stress fluids and in Newtonian materials, under two conditions: (i) if $\alpha_{1}(\phi), \alpha_{2}(\phi)$ and $g(\phi)$ are the same in the yield stress fluid as in a Newtonian material, i.e., if the microstructure is similar in both cases; and (ii), if the yield stress suspension is sheared in the whole gap of the Couette geometry, which cannot be the case near the yielding transition, where shear localization occurs (see Sec. IV A): in such case, the volume fraction in the unsheared region cannot change.

Note that this result cannot be generalized to other flows. Indeed, it is due to (i) the proportionality between the particle normal stresses and the suspension shear stress for both suspending fluids, which implies that the particle stress balance equation has the same form when expressed as a function of the suspension shear stress for both suspending fluids, and (ii) the independence of the shear stress profile on the material behavior in a concentric-cylinder Couette flow. Both equations being independent of the fluid behavior finally implies that the particle volume fraction at equilibrium is the same in both fluids (and in any nonlinear fluid in which the same approach can be conducted). One would thus expect the same features in a Poiseuille flow (as long as the plug region is of negligible extent in the yield stress suspension), where the shear stress profile is also independent of the fluid behavior, but not, e.g., in a parallel plate geometry.

\section{Materials and methods}

\section{A Materials}

Model suspensions of particles in a yield stress fluid are prepared by suspending monodisperse spherical particles in a concentrated emulsion (Tab. 1).

Batches of $2 \mathrm{l}$ of oil-in-water emulsion are prepared by dispersing a $100 \mathrm{~g} / \mathrm{l}$ water solution of $\mathrm{CaCl}_{2}$ in a solution of Span 80 emulsifier $(7 \%)$ in dodecane oil at $6000 \mathrm{rpm}$ with a Silverson 


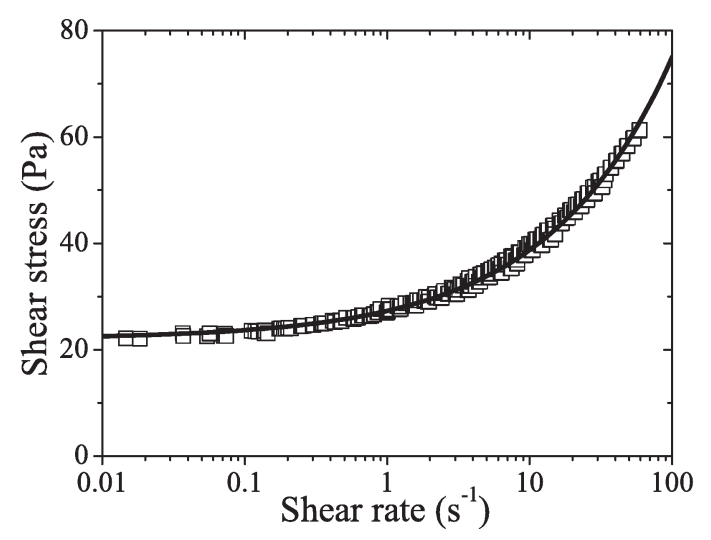

Figure 3: Flow curve of a concentrated emulsion used as a suspending fluid to prepare the suspensions. Data obtained with the help of MRI methods (see Sec. IIIC) in a wide gap concentric-cylinder Couette geometry. The line is a fit to a Herschel-Bulkley equation $\tau=$ $\tau_{y}+\eta_{H B} \dot{\gamma}^{n}$ with $\mathrm{n}=0.5, \tau_{y}=22 \mathrm{~Pa}, \eta_{H B}=5.3 \mathrm{~Pa} . \mathrm{s}^{0.5}$.

L4RT mixer. The average droplet size is $1 \mu \mathrm{m}$ (dispersity: 20\%). The droplet volume fraction is set at $72 \%$. The emulsion density is $\rho=1.01 \mathrm{~g} . \mathrm{cm}^{-3}$. This material is a simple yield stress fluid (Mason et al., 1996; Ovarlez et al., 2008, 2013), i.e. it does not show shear banding nor thixotropy. The flow curve of one of batches is shown Fig. 3. It is well fitted to a HerschelBulkley law of index $n=0.5: \tau=\tau_{y}+\eta_{H B} \dot{\gamma}^{0.5}$, with a yield stress $\tau_{y}=22 \mathrm{~Pa}$ and a consistency $\eta_{H B}=5.3 \mathrm{~Pa} \cdot \mathrm{s}^{0.5}$. From batch to batch, the reproducibility on the value of the rheological properties is not very good, of order $\pm 20 \%$. In the following, when dealing with suspensions, only dimensionless data are provided to quantify the impact of the particles on their rheology: the rheological properties of each suspension are then divided by those of its interstitial emulsion.

In principle, a model suspension could be prepared by dispersing any kind of particle in the yield stress fluid, as long as scale separation is ensured. Indeed, Mahaut et al. (2008a) have observed the same behavior for glass or polystyrene particles dispersed in concentrated emulsions. However, sedimentation always occurs during flows of density-mismatched suspensions, even those that are stable at rest (Ovarlez et al., 2010, 2012). This is illustrated in Fig. 4, where vertical volume fraction profiles of a suspension of $275 \mu \mathrm{m}$ glass particles (density 2.5) in a concentrated emulsion in a concentric-cylinder Couette geometry are shown. The material is stable at rest: elastic forces exerted by the yield stress fluid are able to counterbalance the net gravity force, consistent with the theoretical yield criterion $\tau_{y} / \Delta \rho g d \geq 1 / 21$ (Beris et al., 1985; Tabuteau et al., 2007). By contrast, when flow is enforced, sedimentation occurs: there are no more particles on top of the material, and particles accumulate at the bottom of the geometry. The sedimentation velocity in the dilute regime has been shown to be of order $0.1 \Delta \rho g d^{2} /(\tau(\dot{\gamma}) / \dot{\gamma})$ when a yield stress fluid is sheared at a $\dot{\gamma}$ shear rate (Ovarlez et al., 2012). In the yield stress fluid we study sheared at $\dot{\gamma}=50 \mathrm{~s}^{-1}$, it is thus expected to be of order $0.1 \mathrm{~mm} . \mathrm{s}^{-1}$ for $275 \mu \mathrm{m}$ glass beads, which would make it impossible to study the flows of a homogeneous material far from yielding.

For quantitative studies of flow properties, this artefact should thus be avoided, even with local methods such as MRI methods where the local shear rate is measured in the locally homogeneous material: the torque still is an average over the whole height. Small glass particles, for which settling would be slower, cannot be used because we need the particle diameter to 


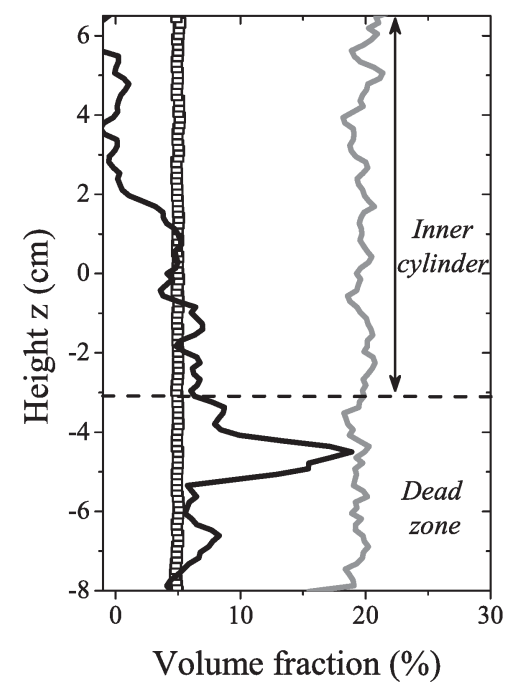

Figure 4: Vertical volume fraction profiles observed with MRI techniques (see Sec. IIIC) in the gap of a concentric-cylinder Couette geometry in a $5 \%$ suspension of $275 \mu \mathrm{m}$ glass beads in a concentrated emulsion of yield stress $\tau_{y}=8.5 \mathrm{~Pa}$, after a $24 \mathrm{~h}$ rest (empty squares) and after 15 min of shear at $\overline{\dot{\gamma}}=4 \mathrm{~s}^{-1}$ (black line), and in a $20 \%$ suspension of $250 \mu \mathrm{m}$ polystyrene beads in a concentrated emulsion of yield stress $\tau_{y}=20 \mathrm{~Pa}$ after $1 \mathrm{~h}$ of shear at $\overline{\dot{\gamma}}=30 \mathrm{~s}^{-1}$ (grey line). Data for the glass beads extracted from Ovarlez et al. (2012).

be much larger than the droplet diameter $(1 \mu \mathrm{m})$ to ensure that the emulsion is 'seen' as a continuous material (a yield stress fluid) by the particles (Mahaut et al., 2008a). We have thus chosen to use polystyrene particles $(d=250 \mu \mathrm{m})$, whose density (1.05) is close to that of the concentrated emulsion; this reduces the sedimentation velocities in the sheared material by a factor 40 as compared to the glass particles. This is confirmed by volume fraction measurements: a suspension sheared during $1 \mathrm{~h}$ at a $30 \mathrm{~s}^{-1}$ average shear rate remains homogeneous along the vertical direction (Fig. 4), which makes it possible to study the flow behavior of the suspension.

The suspension used in the rheo-X-ray microtomography experiments (Sec. IIID) has a slightly different composition from that studied in the other experiments, to allow for a good compatibility with the X-ray setup. The polystyrene beads diameter is $140 \mu \mathrm{m}$ (dispersity: 5\%). Iodine is added in the water phase of the emulsion to ensure a good contrast between the particles and the concentrated emulsion in the X-ray images ( $\mathrm{NaI}$ thus replaces the $\mathrm{CaCl}_{2}$ used in the other prepared emulsions). The emulsion is composed of droplets of a water solution of sodium iodide dispersed at a $77.5 \%$ volume fraction in a solution of Span 80 emulsifier in dodecane oil. Its rheological behavior is well fitted to a Herschel-Bulkley behavior $\tau=\tau_{y}+\eta_{H B} \dot{\gamma}^{0.5}$ with $\tau_{y}=26 \mathrm{~Pa}$ and $\eta_{H B}=5.1 \mathrm{~Pa} . \mathrm{s}^{0.5}$, close to that of the emulsions used in the rheo-NMR experiments.

As in Mahaut et al. (2008a), a suspension is prepared by simply mixing the particles and the fluid in a cup with a spatula, with the goal of achieving a mixing close to chaotic mixing. With this procedure, we aim to prepare a material that is homogeneous and isotropic. Homogeneity is confirmed by the MRI measurements (see below). Isotropy is confirmed by X-ray microtomography (this will be discussed in Sec. IV C). 


\section{B Rheometry}

Since we deal with suspensions of large noncolloidal particles, we need to use a geometry with a wide gap to ensure that the properties of a continuous medium (the suspension) are measured. A geometry classically used to study the flows of such suspensions is the vane-in-cup geometry (Koehler et al., 2006; Martínez-Padilla and Rivera-Vargas, 2006; Jau and Yang, 2010), which is supposed to avoid wall slip (Keentok, 1982; Dzuy and Boger, 1983; Saak et al., 2001): it is assumed that the material in the gap of the geometry is sheared by the (same) material that is trapped between the blades. However, Ovarlez et al. (2011) have shown that a thin layer made of the pure interstitial yield stress fluid appears quickly at the interface between the sheared material and that trapped between the blades. This layer acts as a slip layer, leading to shear rate overestimations; moreover, flows are localized near the tool (and thus, in the depleted layer) at low shear rates, which makes one underestimate the suspension yield stress.

We have thus chosen to use a wide gap concentric-cylinder Couette geometry of inner radius $R_{i}=4.1 \mathrm{~cm}$ and gap $1.9 \mathrm{~cm}$, and inner cylinder height $H=11 \mathrm{~cm}$. In order to avoid wall slip (Coussot, 2005), we glue sandpaper on the walls. Since the shear stress distribution is inhomogeneous in this geometry, it can be expected that flow causes particle migration towards the outer cylinder as in viscous suspensions (Leighton and Acrivos, 1987b; Phillips et al., 1992). To take these possible inhomogeneities into account, we need to use techniques that provide the value of both the local particle volume fraction and the local shear rate (Ovarlez et al., 2006).

\section{Rheo-NMR}

The Couette cell is inserted in a MRI scanner, and proton NMR (Callaghan, 1991, 1999) is used as a non-intrusive technique in order to get measurements of the local material velocity and of the local particle concentration inside the gap. Experiments are performed with a $0.5 \mathrm{~T}$ proton MRI scanner (Bruker Avance 24/80 DBX) operating at $20 \mathrm{MHz}$. It is equipped with a birdcage rf coil (20 cm inner diameter) and a 3D gradient system (BGA26-Bruker). We use a home-made NMR-compliant rheometer fully described in Raynaud et al. (2002).

All data are averaged over $4 \mathrm{~cm}$ in the vertical direction and $1 \mathrm{~cm}$ in the azimuthal direction, and measured with a resolution of $110 \mu \mathrm{m}$ in the radial direction. We checked the homogeneity of the velocity and concentration profiles along the vertical direction in the gap, which justifies averaging data. Note that in the sequences we use, only NMR signal originating from the hydrogen nuclei belonging to the liquid phase of the sample is recorded (relaxation in the PS particles is too fast to be observable with our setup: the particle do not give any signal).

Azimuthal velocity profiles $V(r)$ are obtained with a combination of a spin-echo-spin-warp imaging technique and a phase-encoded velocimetry (Callaghan, 1991, 1999); more details are found in Raynaud et al. (2002); Rodts et al. (2004, 2010). A single velocity measurement may take as little as $1 \mathrm{~s}$. Particle concentration profiles $\phi(r)$ are obtained with a spin-echo sequence (Callaghan, 1991); NMR data are recorded during a readout gradient and Fourier transformed so as to get information about hydrogen density in the liquid phase along the radial direction; more details can be found in Ovarlez et al. (2006). A concentration profile is obtained in 3 min.

In the experiments, we control the rotational velocity $\Omega$ of the Couette cell inner cylinder, and we measure the torque $T(\Omega)$ with a Bohlin C-VOR 200 rheometer.

The local shear rate $\dot{\gamma}(r)$ at a radial position $r$ in the gap can be deduced from $V(r)$ as

$$
\dot{\gamma}(r)=V(r) / r-\partial_{r} V(r)
$$

The derivative $\partial_{x} f$ with respect to coordinate $x$ of experimental data $f\left(x_{i}\right)$ measured at regularly spaced positions $x_{i}$ was here computed as: $\partial_{x} f\left(x_{i}\right)=\left[f\left(x_{i+1}\right)-f\left(x_{i-1}\right)\right] /\left[x_{i+1}-x_{i-1}\right]$. 
The stress distribution $\tau(r)$ within the gap is obtained from Eq. 37 which yields

$$
\tau(r)=\frac{T}{2 \pi r^{2} H}
$$

when the material is homogeneous along the vertical direction.

If the material is homogeneous in the radial direction, local data $(\tau(r, \Omega), \dot{\gamma}(r, \Omega))$ measured at various $r$ and various $\Omega$ can finally be combined to obtain the constitutive law $\tau(\dot{\gamma})$ consistent with the observed flows (more details about this reconstruction technique can be found in Ovarlez et al. (2008)). Local $\tau(\dot{\gamma})$ data measured with this method in a (pure) concentrated emulsion are displayed in Fig. 3. Note that, as Eqs. 41 and 42 are valid whatever the boundary conditions are, this analysis is not affected by a possible wall slip.

Of course, for a homogeneous simple yield stress fluid, this analysis is not necessary and may seem uselessly complex since a simple rheometer with appropriate procedures provides the same result (Ovarlez et al., 2013). However, in the case of suspensions, this methodology can become indispensable. Indeed, as already mentioned, particles tend to migrate toward the outer cylinder in a concentric-cylinder Couette flow, which can lead to strong particle volume fraction inhomogeneities (Phillips et al., 1992; Morris and Boulay, 1999; Ovarlez et al., 2006). The kinetics of this migration phenomenon is slow at low volume fraction, but gets more and more rapid as the volume fraction increases. This is also at high particle volume fraction that the impact of inhomogeneities is the strongest since rheological properties diverge at the approach of $\phi_{\text {div }}$; data obtained with a rheometer only may then lead to gross underestimations of these rheological properties (Ovarlez et al., 2006; Hafid et al., 2015). It can take as little as a few units of strains for the material to become inhomogeneous at high volume fraction (Ovarlez et al., 2006). It may thus be impossible to characterize the flow properties of the homogeneous material. E.g., for $50 \%$ of particles in the yield stress fluid, we found that a steady inhomogeneous volume fraction profile is reached after less than 1000 units of strain.

It is worth noting that we found that, as long as all the material is sheared during migration ${ }^{4}$, steady-state volume fraction profiles do not depend on the rotation velocity $\Omega$, which is consistent with the theoretical prediction Eq. 40. In such case, the above analysis to obtain the constitutive behavior from local measurements can be conducted again. Indeed, since $\phi(r)$ is independent of $\Omega$, a change of variables can be performed between radial position $r$ and $\phi$. A local stress/strainrate relationship $\tau(\dot{\gamma}, \phi)$ at fixed and well-defined volume fraction $\phi$ is then obtained by collecting all measurements of local stress $\tau(r)$ and shear rate $\dot{\gamma}(r)$ as above, for varying $\Omega$, but for a single fixed $r$. More details on this methodology can be found in Ovarlez et al. (2006); Fall et al. (2010, 2015). The main difference with the method used to characterize the pure emulsion is that now, measurements for a single value of $r$ are collected together to characterize a given $\phi$. In practice, to ensure that enough data are collected, we combined together data obtained in a region of the gap where volume fraction variation is limited to $\pm 0.75 \%$.

In this paper, instead of trying to avoid migration, which would have not been possible above $40 \%$ volume fraction, we chose to ensure that a steady-state is reached. We have thus first presheared the material at a rotation velocity $\Omega=100 \mathrm{rpm}$ during 1 hour in all cases. The rotation velocity was then decreased step by step from 100 to $1 \mathrm{rpm}$ in order to collect data at various local shear rates with the procedure described above; this procedure (decreasing sweep) has the advantage of avoiding artefacts associated to transient shear banding behavior (Ovarlez et al., 2013).

\footnotetext{
${ }^{4}$ This is true unless a dead zone, where $\dot{\gamma}=0$, is present during the preshear. In such case, the material remains homogeneous in the dead zone, the size of which depends on the applied rotational velocity. In the experiments presented in this paper, we ensured that the whole gap is sheared during the preshear.
} 


\section{Rheo-X-ray microtomography}

A few experiments have been performed to characterize the suspensions' microstructure, i.e., the spatial distribution of particles in the suspensions. There is no room to detail the technique and the results here; an in-depth study will be presented in a forthcoming publication. We only provide below the main characteristics of the setup; additional details can be found in Deboeuf et al. (2013).

We have used a parallel plate geometry (of gap $H_{p p}=2 \mathrm{~mm}$ and radius $R_{p p}=1 \mathrm{~cm}$ ) coupled to a X-ray microtomograph (Maire et al., 2001), which allows us to get 3D images of suspensions and to determine the particles' position. The measurements are conducted with an Ultratom scanner from RXSolutions. The spatial resolution (voxel size of the images) is $12 \mu \mathrm{m}$. To characterize the suspension microstructure, a given shear history is imposed to the suspension in the rheometric cell, and then stopped as long as needed for the complete scanning of the sample (about 1 hour). The suspension microstructure is then assumed to be the same just before and after the interruption of shear thanks to the fluid yield stress, although slight relaxation of the interstitial fluid can be expected below the yield stress (Mohan et al., 2015). The absence of blur in the images confirms that the particles do not move at rest during the $1 \mathrm{~h}$ scan.

The suspension studied in these experiments has a slightly different composition from that studied in the rheo-NMR experiments (see Sec. III A), to allow for a good compatibility with the setup; it has, nevertheless, similar rheological properties. In particular, the polystyrene beads diameter is $140 \mu \mathrm{m}$ to ensure that the gap is more than 10 particles wide. Moreover, the contrast between the particles and the concentrated emulsion in the X-ray images is increased by the addition of iodine in the water phase of the emulsion. X-ray absorption being different between the particles and the fluid, the particles are easily identified; the spherical shape of the particles then allows us to determine the positions of particle centers with a subvoxel resolution of $1 \mu \mathrm{m}$. Various quantities characterizing the particle distribution in space can then be computed to describe the suspension microstructure. We will focus on pair distribution functions in Sec. IV C.

Since shear is inhomogeneous in a parallel plate geometry, we restrict the analysis of particle distribution to the region of the gap of radial position $0.6 R_{p p} \leq r \leq 0.84 R_{p p}$; in this region, shear is roughly homogeneous, and the microstructure can be studied as a function of the average shear rate $\dot{\gamma}=0.72 \Omega R_{p p} / H_{p p}$ by changing the rotation velocity $\Omega$ of the upper plate of the geometry.

\section{Flow behavior}

\section{A Main characteristics of the flows}

The steady velocity profiles observed in a pure emulsion and in the same emulsion filled with $30 \%$ of particles are displayed in Figs. 5a and b. These profiles are typical of shear-thinning yield stress fluids in a concentric-cylinder Couette geometry and have already been described and commented in detail elsewhere (Coussot, 2005; Ovarlez et al., 2008). They can be explained thanks to the $1 / r^{2}$ decrease of the shear stress in the gap of the geometry. These profiles are curved at high shear rate, due to shear-thinning. They get more and more curved as the velocity decreases and a dead zone appears near the outer cylinder at the lowest velocities; this is due to the existence of a yield stress, the shear stress being equal to the yield stress inside the gap in these last cases.

At first sight, there is no qualitative change in the behavior when adding particles. This is consistent with the prediction that a suspension of particles in a Herschel-Bulkley material 
(a)

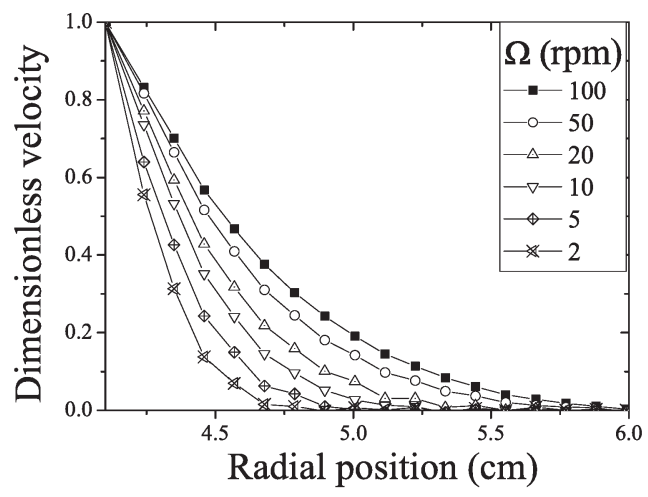

(c)

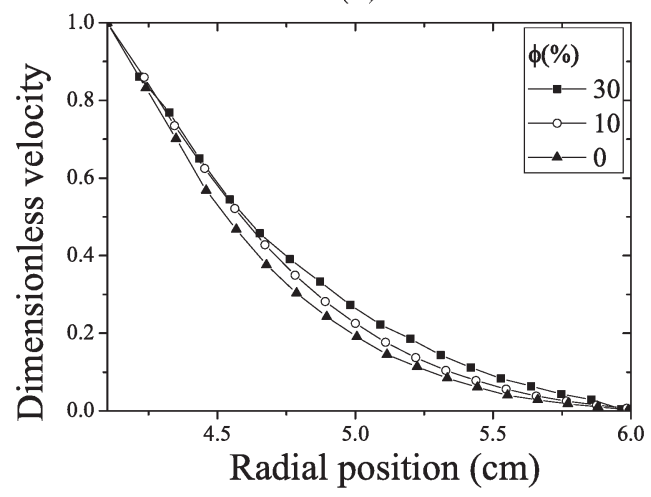

(b)

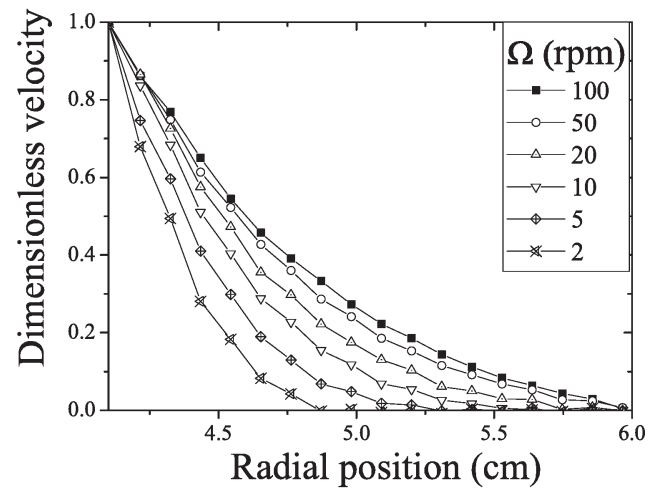

(d)

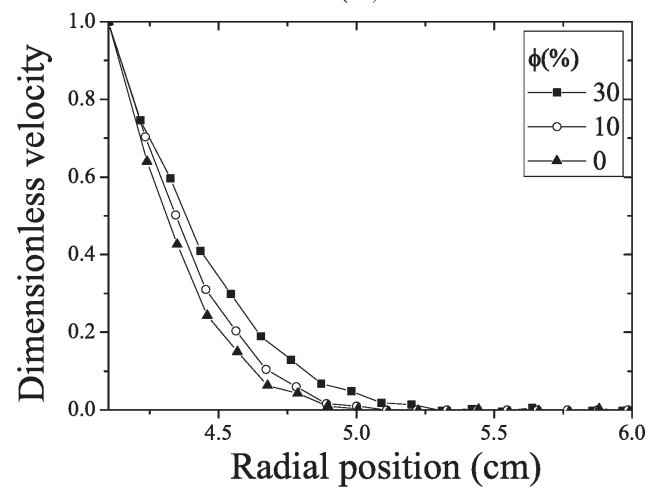

Figure 5: (a) Velocity profiles of a pure emulsion in the rheo-NMR Couette geometry at various rotation velocities $\Omega$ (see legend). (b) Velocity profiles of the same emulsion filled with $30 \%$ of particles. (c) Velocity profiles at $\Omega=100 \mathrm{rpm}$ for the pure emulsion, and the same emulsion filled with $10 \%$ and $30 \%$ of particles. (d) Same as (c) at $\Omega=5 \mathrm{rpm}$.

behaves as a Herschel-Bulkley material. Looking more closely to the data, one observes that the velocity profiles are shifted toward the outer cylinder when adding particles and seem less curved. This is better seen in Figs. $5 \mathrm{c}$ and d, where profiles obtained at a same velocity are directly compared. It is in particular observed that, for a same rotation velocity, in the presence of shear localization, the size of the sheared zone increases when adding particles. For HerschelBulkley materials of a given index $n$, this can be interpreted as being due to a sharper increase with $\phi$ of the viscous stress $\eta_{H B} \dot{\gamma}^{n}$ as compared to the yield stress $\tau_{y}$. The fact that profiles are less curved at high rotation velocity has the same interpretation: when $\eta_{H B} \dot{\gamma}^{n} / \tau_{y}$ increases, the velocity profiles get closer from the asymptotic profile, which is that of a shear-thinning material of power-law index $n$. Our observations are thus consistent with one of the predictions of the model, which is that $\eta_{H B}(\phi) \dot{\gamma}^{n} / \tau_{y}(\phi)$ is an increasing function of $\phi$. Indeed, from Eqs. 21 and $22, \frac{\eta_{H B}(\phi) \tau_{y}(0)}{\eta_{H B}(0) \tau_{y}(\phi)}=\frac{g(\phi)^{n / 2}}{(1-\phi)^{n / 2}}$. This is an interesting feature of these suspensions: although their yield stress increases, they are less prone to form dead zones under controlled flow rate; the same is expected to be observed in pipe flows.

In Fig 6, we plot the concentration profiles obtained after $1 \mathrm{~h}$ of shear at $100 \mathrm{rpm}$ in suspensions of volume fraction ranging from 10 to $50 \%$. As far as we can say, these are steady 
(a)

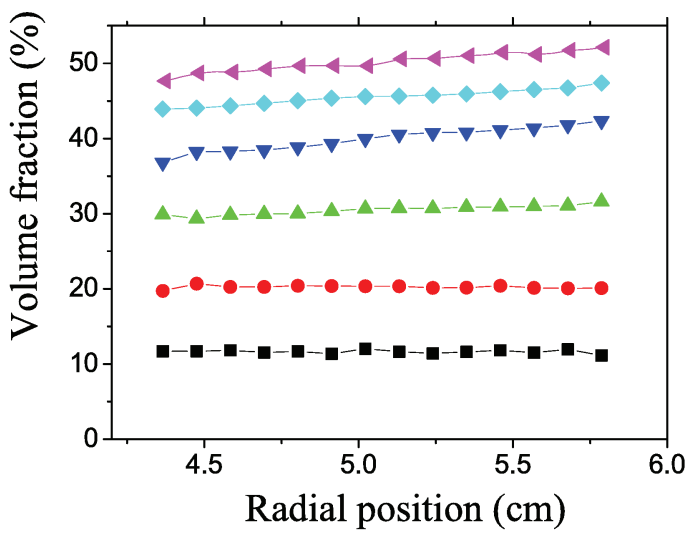

(b)

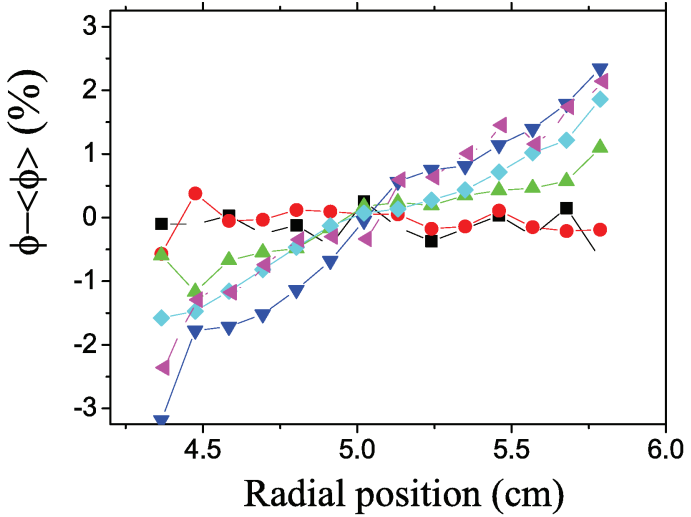

Figure 6: (a) Concentration profiles measured in the rheo-NMR Couette geometry in suspensions of concentration (from bottom to top) 10\%, 20\%, 30\%, 40\%, 45\%, and 50\%. (b) Same data as in (a): the deviation of concentration $\phi$ from its average $\langle\phi\rangle$ is shown for each suspension.

concentration profiles, and they do not change when subsequently changing $\Omega$ : we checked that after performing the velocity sweep to get velocity profiles, these profiles did not change. No observable migration is obtained below a $20 \%$ volume fraction. On the other hand, significant shear-induced migration from the inner cylinder to the outer cylinder is observed for volume fractions $\geq 30 \%$. These profiles will be analyzed further in Sec. IV F. At this stage, this observation justifies the use of advanced MRI techniques to obtain the constitutive law of these suspensions. Indeed, at the highest concentrations, volume fraction variations within the gap are of the order of $5 \%$. Such variations have a priori a significant impact on the suspension flow properties; e.g., from Fig. 2, it is seen that the linear response $g(\phi)$ increases by a factor of order 2 to 3 between $45 \%$ and $50 \%$. To analyze the behavior of the suspension, in each case, following the steps presented in Sec. IIIC, we limit the analysis of the local shear rate $\dot{\gamma}(r)$ and shear stress $\tau(r)$ data to zones where $\phi(r)$ variation is less than $\pm 0.75 \%$. E.g., for the $30 \%$ volume fraction suspension, only data obtained in the zone $4.7 \mathrm{~cm}<r<5.4 \mathrm{~cm}$ are combined to get $\tau(\dot{\gamma})$, which characterizes a material of volume fraction $\phi=30 \pm 0.5 \%$. For the $50 \%$ volume fraction suspension, data combined in the zone $4.4 \mathrm{~cm}<r<4.7 \mathrm{~cm}$ characterize the material of volume fraction $\phi=48 \pm 0.75 \%$, whereas data combined in the zone $4.9 \mathrm{~cm}<r<5.4 \mathrm{~cm}$ characterize the material of volume fraction $\phi=49 \pm 0.75 \%$. The results of this analysis are presented below.

\section{B Constitutive behavior}

In Fig. 7 , we present the local constitutive behavior $\tau(\dot{\gamma})$ obtained from the analysis of MRI data for a concentrated emulsion, and for suspensions of $10 \%$ and $30 \%$ particles in the same emulsion, together with fits of Herschel-Bulkley laws to the data.

It is first observed that the yield stress increases only slightly when adding particles, whereas a significant increase of the viscous effects is visible. This confirms our first observations based on the velocity profiles only. To analyze quantitatively the data, a reference to a model is necessary. Since the Herschel-Bulkley law provides a good fit to the emulsion behavior, we chose to analyze all of our data in the framework of this model. In Fig. 7, we observe that the three materials can be fairly modeled by a Herschel-Bulkley law of same index $n$ (here, $n \simeq 0.5$ ) in all 


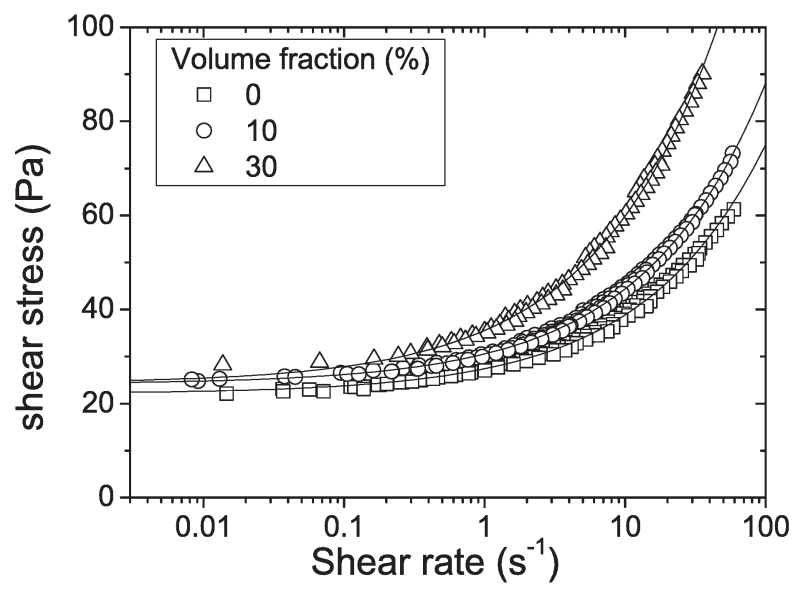

Figure 7: Local constitutive behavior $\tau(\dot{\gamma})$ measured using MRI techniques in a concentrated emulsion, and in suspensions of particles in the same emulsion at volume fractions $\phi=10 \%$ and $30 \pm 0.5 \%$. The lines are fits of a Herschel-Bulkley behavior $\tau_{y}+\eta_{H B} \dot{\gamma}^{0.5}$ to the data with, from bottom to top, $\tau_{y}=22.1 \mathrm{~Pa}$ and $\eta_{H B}=5.3 \mathrm{~Pa} . \mathrm{s}^{0.5}, \tau_{y}=24.1 \mathrm{~Pa}$ and $\eta_{H B}=6.4 \mathrm{~Pa} . \mathrm{s}^{0.5}$, $\tau_{y}=24.3 \mathrm{~Pa}$ and $\eta_{H B}=11.25 \mathrm{~Pa} . \mathrm{s}^{0.5}$.

cases, as predicted by the theory. This is a general feature, observed for all concentrations.

A definitive conclusion on the fact that the same index characterizes all materials would require data at stresses much higher than the yield stress for a closer inspection of the asymptotic behavior. Letting $n$ as a free parameter, values between 0.44 and 0.52 were actually found; nevertheless, fixing $n=0.5$ provided fits with coefficient of determination $R^{2}>0.99$ in all cases. At this stage, we just choose to fix the value of $n$ in order to be able to compare the values of the consistencies $\eta_{H B}$ obtained at various $\phi$; the choice of $n=0.5$ is made in agreement with the literature of concentrated emulsions (Mason et al., 1996; Ovarlez et al., 2008; Seth et al., 2011) and has theoretical justifications for such materials (Seth et al., 2011).

In the following, we study the evolution with the particle volume fraction $\phi$ of the dimensionless consistency $\eta_{H B}(\phi) / \eta_{H B}(0)$ and of the dimensionless yield stress $\tau_{y}(\phi) / \tau_{y}(0)$ extracted from the fit to the data of a Herschel-Bulkley behavior with $n=0.5$.

\section{Consistency}

In Fig. 8, we observe that the consistency significantly increases with the volume fraction, this increase being of a factor of order 5 for $\phi=50 \%$. The observed evolution is in very good agreement with the prediction of Eq. 32, which is based on the linear response of suspensions as observed by Thomas (1965) and Sierou and Brady (2002). By contrast, there is a strong mismatch between our data and the predictions of Eq. 34, which is based on the linear behavior observed by Zarraga et al. (2000) and Dai et al. (2013). This suggests that our suspension microstructure is similar to that of suspensions of low friction particles in Newtonian media, as this corresponds to the situation of the Sierou and Brady (2002) simulations; indeed, as discussed in Sec. II B 2, it seems that the lowest possible $g_{\text {aniso }}(\phi)$ are obtained when interparticle friction is negligible (Sierou and Brady, 2002; Gallier et al., 2014).

Note that the equation used here for $g_{\text {aniso }}(\phi)$ (Eq. 29) was validated only up to $50 \%$ in Fig. 2; it is known, however, that the asymptotic behavior of $g_{\text {aniso }}(\phi)$, at the vicinity of jamming, is 


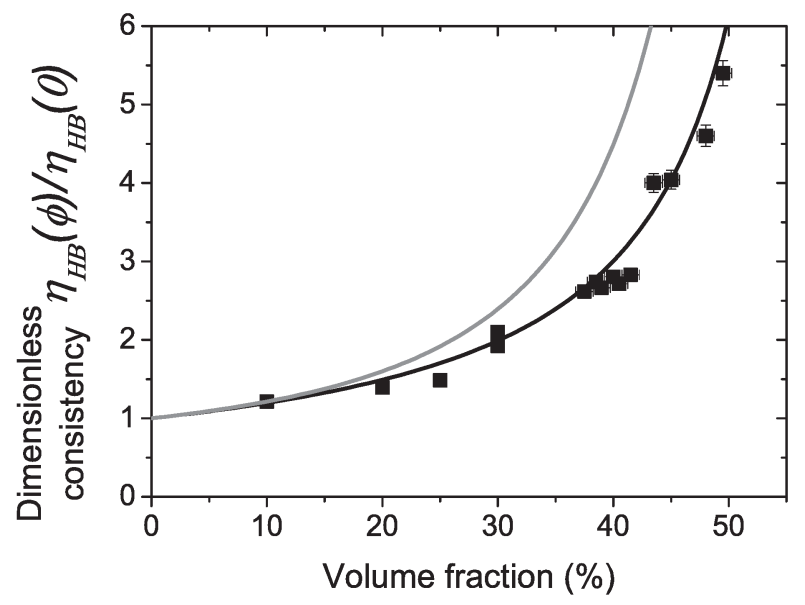

Figure 8: Dimensionless consistency $\eta_{H B}(\phi) / \eta_{H B}(0)$ versus volume fraction $\phi$ for all the studied suspensions of particles in concentrated emulsions, extracted from a fit of the $\tau(\dot{\gamma})$ data to a Herschel-Bulkley law of index $n=0.5$. The black line is the model (Eq. 32) based on the linear behavior observed by Thomas (1965) and Sierou and Brady (2002); the grey line is the model (Eq. 34) based on the linear behavior observed by Zarraga et al. (2000) and Dai et al. (2013).

different from Eq. 27 (Ovarlez et al., 2006; Boyer et al., 2011b). Our model for the consistency (Eq. 32) is thus expected to be valid only in the range of volume fraction we have studied, from 0 to $50 \%$. The behavior for $\phi_{\operatorname{div}}>\phi>45 \%$ has been investigated in a recent paper (DagoisBohy et al., 2015), published while our paper was under review. The authors have added a free parameter to our relationship (Eq. 12) between the local shear rate and the macroscopic shear rate in order to fit their data to our model; they report that, if the $g_{\text {aniso }}(\phi)$ they measure in a viscous suspension is correct, then $\overline{\dot{\gamma}}_{\text {local }}(\phi) \simeq 2 \times \dot{\gamma} \sqrt{g_{\text {aniso }}(\phi) /(1-\phi)}$, which is not at all justified in the theoretical approach derived in Sec. II. As pointed out in Sec. II B 2, this might be due to the choice of PEG as an interstitial fluid to measure $g_{\text {aniso }}(\phi)$; another choice would have provided a different $g_{\text {aniso }}(\phi)$. This might also come from two limitations of our theoretical approach: direct interparticle dissipation was neglected, and the estimate chosen for $\overline{\dot{\gamma}}_{\text {local }}(\phi)$ (Eq. 5) might not be relevant in the very dense regime, where most of viscous dissipation occurs in the small volume occupied by the fluid between close particles. The behavior at the approach of $\phi_{\text {div }}$ thus remains to be investigated theoretically; it will not be discussed further here.

\section{$2 \quad$ Yield stress}

We now turn to the analysis of the yield stress data. In Fig. 9, we observe a very moderate increase of $\tau_{y}(\phi)$ with the volume fraction $\phi$, this increase being of less than $10 \%$ for $\phi \leq 40 \%$, and the maximum observed increase being of a factor 1.6. The scattered data at the highest volume fraction investigated, where the yield stress at $50 \%$ seems to be lower than the yield stress at $45 \%$, is likely due to the fact that yield stresses are here obtained from a fit to $\tau(\dot{\gamma})$ data with a limited amount of data at low shear rates. Indeed, as discussed in Sec. IV A, $\eta_{H B} \dot{\gamma}^{n} / \tau_{y}$ is an increasing function of $\phi$; an accurate determination of $\tau_{y}$ thus requires stress measurements at lower and lower $\dot{\gamma}$ as $\phi$ is increased. At the lowest rotation velocity reachable by the motor $(1 \mathrm{rpm})$, there is no shear localization in the $50 \%$ suspension, and the lowest value of the local shear rate in the gap is of order $0.1 \mathrm{~s}^{-1}$, which is not sufficient to describe accurately the yield 


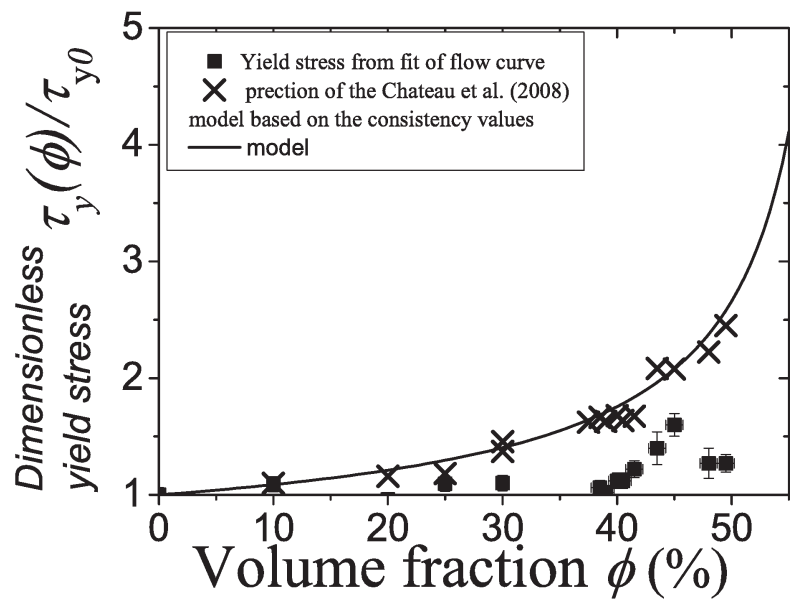

Figure 9: Squares: dimensionless yield stress $\tau_{y}(\phi) / \tau_{y}(0)$ versus volume fraction $\phi$ for all the studied suspensions of particles in concentrated emulsions, extracted from a fit to the $\tau(\dot{\gamma})$ data of a Herschel-Bulkley law of index $n=0.5$. Full line: model (Eq. 31) based on the linear behavior observed by Thomas (1965) and Sierou and Brady (2002). Crosses: dimensionless consistency data plotted as $\left[\eta_{H B}(\phi) / \eta_{H B}(0)\right]^{1 /(n+1)}(1-\phi)^{n /(n+1)}$ with $n=0.5$.

stress plateau. At all other volume fractions, there is shear localization, local shear rates of order $0.01 \mathrm{~s}^{-1}$ are measured (see e.g. Fig. 7), and the yield stress is better estimated.

By contrast with the consistency data, the yield stress values are much lower than expected from the model (Eq. 31). The model can actually be tested independently of the previous results. Indeed, one of the predictions is that there exists an equation (Eq. 23) without any fitting parameter linking the dimensionless consistency and the dimensionless yield stress. To test this prediction, we plot in Fig. 9 the dimensionless consistency data in the form $\left[\eta_{H B}(\phi) / \eta_{H B}(0)\right]^{1 /(n+1)}(1-\phi)^{n /(n+1)}$ with $n=0.5$, which should be equal to $\left(\tau_{y}(\phi) / \tau_{y}(0)\right)$ according to Eq. 23. There is no agreement at all, whereas the $\left[\eta_{H B}(\phi) / \eta_{H B}(0)\right]^{1 /(n+1)}(1-\phi)^{n /(n+1)}$ data match quite well the model for the yield stress (Eq. 31).

A partial conclusion is thus that the model presented in Sec. II fails to predict the flowing behavior of the suspensions in the sense that there is no simple link between their yield stress and their consistency. The consistency is fairly well modeled, but the dependence of the yield stress on volume fraction remains a puzzle. As mentioned in Sec. II A, a key assumption to get Eq. 23 is that the material microstructure does not depend on $\dot{\gamma}$, i.e. that it is similar at low and high shear rate, which is far from being obvious for a nonlinear interstitial fluid. To go one step further, it is necessary to question this assumption. This is the subject of the next section.

\section{Microstructure}

The spatial distribution of particles is known to depend on flow history in suspensions of noncolloidal particles in Newtonian fluids, which has an impact on the material properties (Morris, 2009; Blanc et al., 2011b, 2013). In the case of a nonlinear interstitial fluid, the steady-state particle microstructure under flow can moreover a priori be expected to be a function of the shear rate, which contrasts with the case of a Newtonian interstitial fluid. To investigate this point, as described in Sec. IIID, we use X-ray microtomography to determine the positions of all particle centers for different shear histories in a simple shear flow. 
(a)
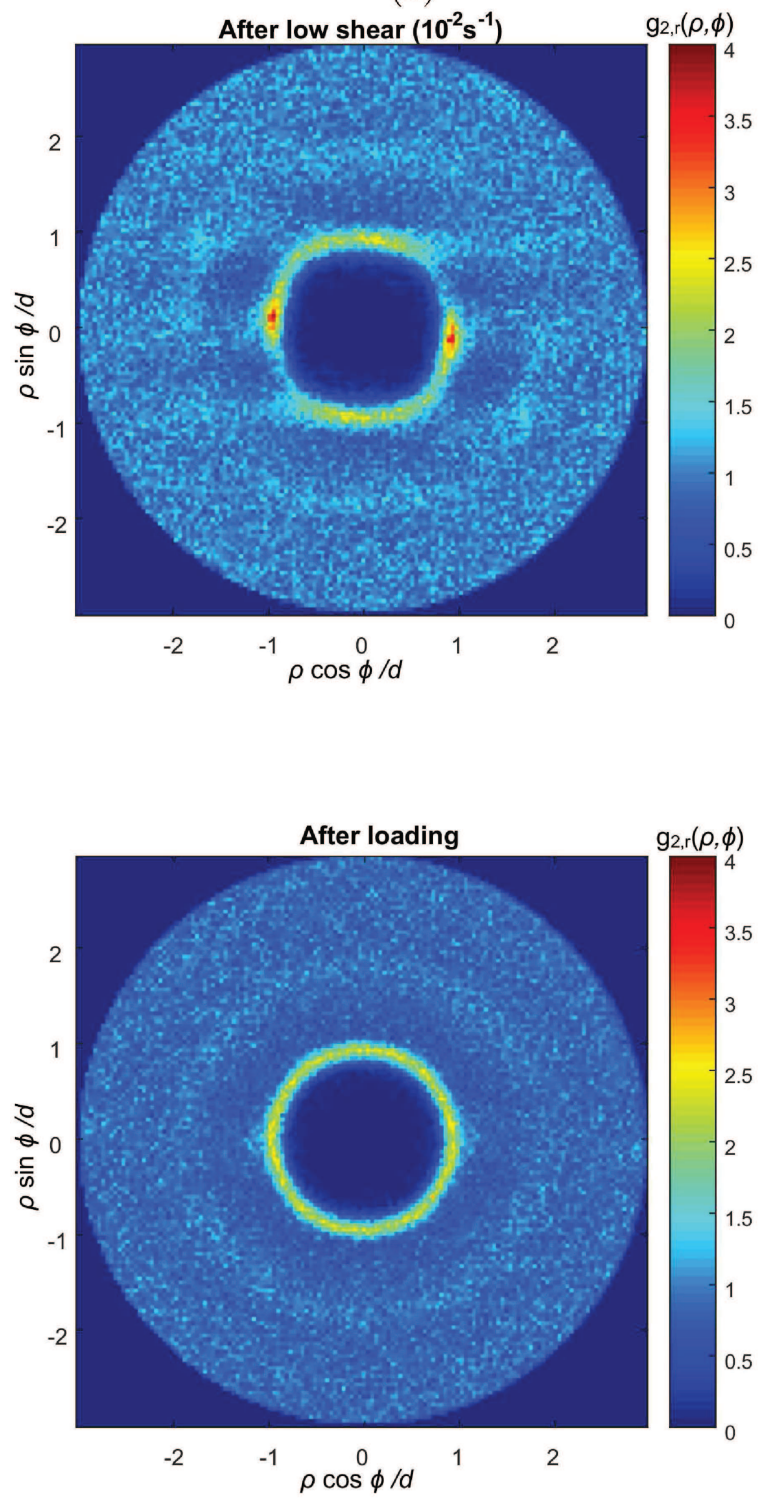

(c) (b)
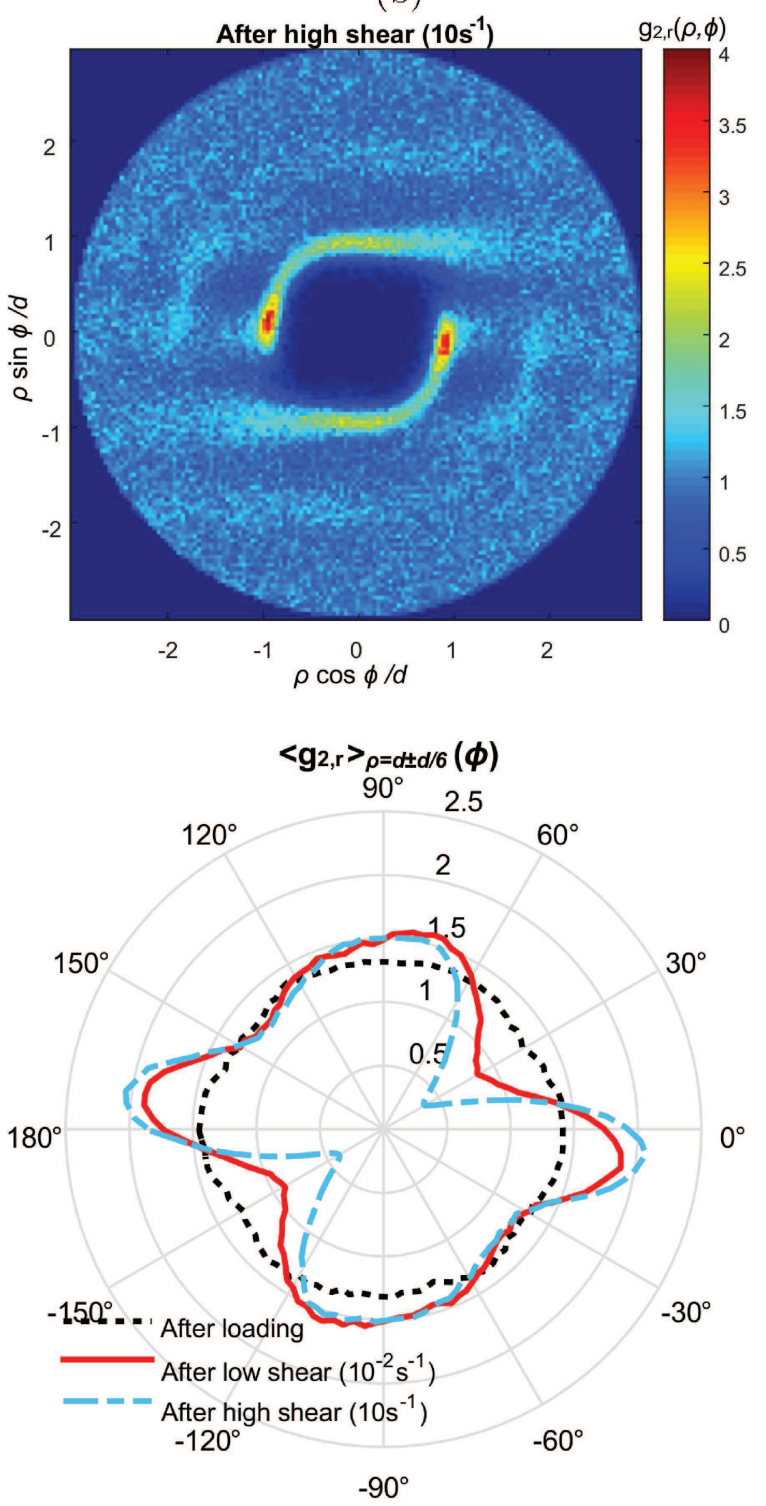

(d)

Figure 10: (a)-(b)-(c) Color maps of pair distribution functions $g_{2, r}(\rho, \phi)$ in the velocity-velocity gradient plane of the simple shear flow imposed locally by a parallel plate geometry for a suspension of $37 \%$ particles in a concentrated emulsion, in three different conditions: (a) after shear at low shear rate $\left(10^{-2} \mathrm{~s}^{-1}\right)$; (b) after shear at high shear rate $\left(10 \mathrm{~s}^{-1}\right)$; and (c) after loading the sample into the geometry. $(\rho, \phi)$ are the polar coordinates of a particle pair in the velocity (horizontal axis)-velocity gradient (vertical axis) plane. (d) Polar plot of the pair distribution function as a function of the pair angle $\phi$ for close neighbors: $g_{2, r}$ is here averaged over pairs of distance $\rho$ equal to $d \pm d / 6, d$ being the particle diameter. The pdfs are computed from the particle positions determined using X-ray microtomography for the different shear histories. 
A relevant quantity to describe the suspension microstructure is the pair distribution function $g_{2}(\vec{r})$ (Morris, 2009). $g_{2}(\vec{r})$ is the probability of finding a pair of particles whose centers are separated by the vector $\vec{r}$ normalized by the mean particle density, so that the asymptotic value of $g_{2}$ for large values of $\|\vec{r}\|$ is $g_{2}(\|\vec{r}\| \rightarrow \infty)=1$. To compute this function, all particles are subsequently chosen to be the 'reference particle', the other particles being the 'test particles', and we count the number of test particles whose centers are separated by $\vec{r}$ from the center of the reference particle. Finally, the total number of particles separated by $\vec{r}$ from a reference particle is normalized to give the pair distribution function $g_{2}(\vec{r})$.

We focus on the microstructure of the suspension in the velocity-velocity gradient plane of the simple shear flow imposed locally by the geometry, through the computation of the statistical pair distribution function $g_{2, r}(\rho, \phi)$, with $\rho$ and $\phi$ the polar coordinates of a pair of particles located in the velocity-velocity gradient plane.

In Fig. 10, steady-state pair distribution functions (pdf) obtained in a suspension of $37 \%$ beads in a concentrated emulsion are displayed; they correspond to three different conditions: (a) after shear at low shear rate $\left(10^{-2} \mathrm{~s}^{-1}\right)$; (b) after shear at high shear rate $\left(10 \mathrm{~s}^{-1}\right)$; and (c) after loading into the rheometer. As expected, the three pdfs are clearly different: the material is isotropic after loading and anisotropic in the plane of shear under flow, and the details of the anisotropy depend on the applied shear rate.

More precisely, after loading, the pdf $g_{2, r}(\rho, \phi)$ depends only on the distance $\rho$, and not on the angular direction $\phi$. We did not study in detail the conditions to get isotropy; we hypothesize that it requires to achieve a mixing close to chaotic mixing when preparing the suspension, and to avoid imposing a significant unidirectional flow to the material before any measurement.

Under flow, $g_{2, r}(\rho, \phi)$ now depends on both $\rho$ and $\phi$. For distances $\rho$ close to the value of the particle diameter, which correspond to near neighbors and possibly to particles in contact, it is observed (Fig. 10d) that $g_{2, r}(\rho, \phi)$ has maximal values for the angles $+170^{\circ}$ and $-10^{\circ}$, and minimal values for the angles $+30^{\circ}$ and $-150^{\circ}$, corresponding, respectively, to an increase of the number of particle pairs roughly aligned with the flow, in the compressional region of simple shear, and to a decrease of the number of pairs in the extensional region. Secondary maxima and minima are observed too. These features are qualitatively similar to what is observed for particles in Newtonian fluids (Morris, 2009; Blanc et al., 2013). We also observe in Figs. 10a and $10 \mathrm{~b}$ a 'tail' of large values of $g_{2, r}$ roughly aligned with the flow direction in the extensional region.

Importantly, the shape of the pdf $g_{2, r}(\rho, \phi)$ depends on the shear rate, which contrasts with the case of interstitial Newtonian fluids: the depletion of particle pairs in close contact in the extensional region is indeed enhanced by high shear rates (Fig. 10d), which is counter-balanced by a growing tail (Fig. 10b). We thus propose to explain qualitatively the failure of Eq. 23 observed in Fig. 9, i.e. the apparent absence of a link between the dynamic yield stress value and the consistency, from the differences in the particle microstructures. Indeed, the consistency value is obtained from the suspension behavior at high shear rate whereas the dynamic yield stress is obtained from the behavior at low shear rate. These two properties thus reflect two different microstructures.

\section{Static yield stress vs dynamic yield stress: macroscopic measurements}

To test further this idea, we have performed additional macroscopic experiments in a parallel plate geometry (of $40 \mathrm{~mm}$ radius and $2 \mathrm{~mm}$ gap) in which we have presheared the suspension at high shear rate; we have then stopped abruptly the rotation and left the material at rest to relax during 5 minutes. With this procedure, we expect the particle microstructure formed 
(a)

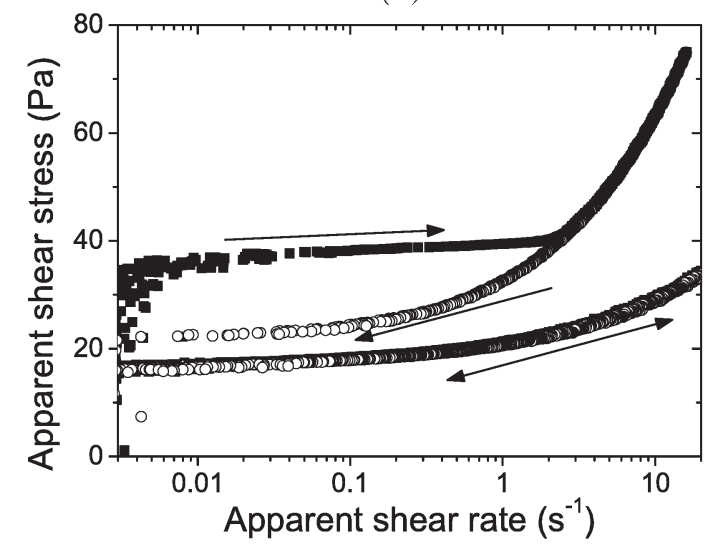

(b)

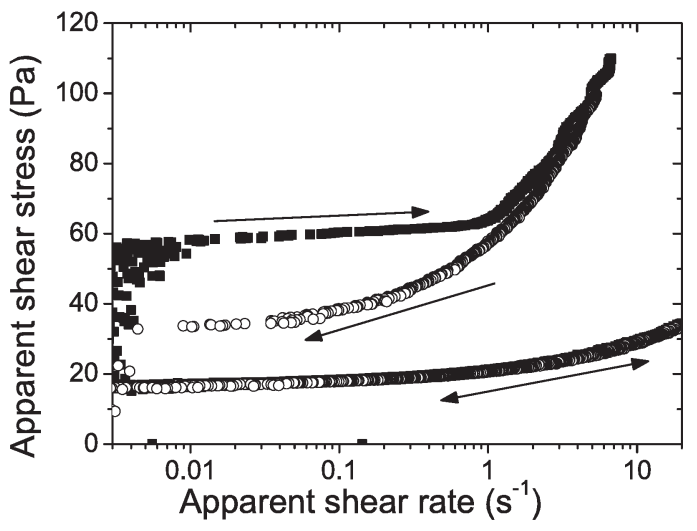

Figure 11: Flow curves measured in a parallel plate geometry: (a) in a concentrated emulsion (lower curves) and in the same emulsion filled with $40 \%$ of particles (upper curves); and (b) in a concentrated emulsion (lower curves) and in the same emulsion filled with $50 \%$ of particles (upper curves). After a 2 min presehar at $10 \mathrm{~s}^{-1}$ and a 5 min rest, the shear stress is ramped up (squares) and down (empty circles) linearly at a 0.5 Pa.s rate.

under shear to be gelled at rest; any measurement performed subsequently should then bear the signature of this microstructure. In particular, if a shear flow at low shear rate is subsequently imposed on the material, a transient stress response is expected before the material reaches a steady-state stress corresponding to the steady-state microstructure characterizing a slow flow. In the following, to account for such possible transient shear-history-dependent effects, we now make the distinction between the static yield stress $\tau_{y, s}$, which is the stress above which flows start, and the dynamic yield stress $\tau_{y, d}$, which is the stress below which flows stop.

After the preshear at high shear rate and the rest period, we have applied a linear shear stress up-ramp to measure $\tau_{y, s}$, followed by a linear down-ramp to measure the flow curve and in particular $\tau_{y, d}$. The results of such macroscopic measurements performed on a concentrated emulsion, and on the same material filled with 40 and $50 \%$ of particles are displayed in Fig. 11.

Whereas the behavior of the pure emulsion does not show any significant dependence on flow history, as classically observed for such simple yield stress fluids (Ovarlez et al., 2013), the suspensions do display a static yield stress $\tau_{y, s}$ much higher than their dynamic yield stress $\tau_{y, d}$ (upper curves of Figs. 11a and b). Indeed, when the shear stress $\tau$ is slowly increased from rest, flow starts abruptly when $\tau$ reaches a value $\tau_{y, s}$ that is roughly 2 times higher than $\tau_{y, d}$; the shear rate $\dot{\gamma}$ then increases rapidly until the steady-state flow curve $\tau(\dot{\gamma})$ is reached. The data subsequently follow reversibly the steady-state flow curve of the suspension during the rest of the up-ramp and the beginning of the down-ramp. During the down-ramp, the data continue following this steady-state flow curve: $\dot{\gamma}$ decreases smoothly when $\tau$ is below the $\tau_{y, s}$, and goes toward zero when $\tau$ approaches $\tau_{y, d}$, below which there is no more flow. It is worth noting that the flow curve $\tau(\dot{\gamma})$ is followed reversibly if the shear stress is ramped-up again just after the down-ramp: in this case, the static yield stress is equal to the dynamic yield stress because the microstructure gelled at rest is that formed under low shear rate.

We thus conclude that the static yield stress is the yield stress of the material which has the microstructure formed during its preparation, whereas the dynamic yield stress is the yield stress of the material whose microstructure is the steady-state microstructure formed under low 


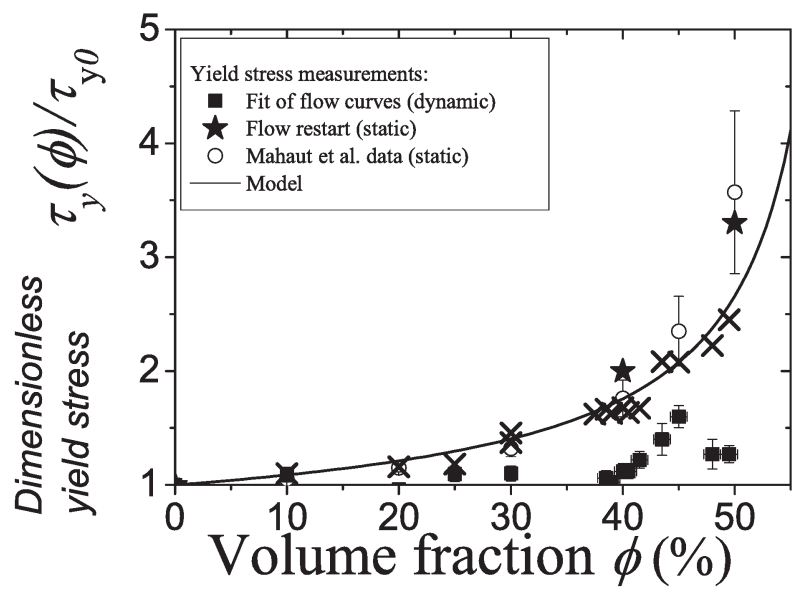

Figure 12: Squares: dimensionless yield stress $\tau_{y}(\phi) / \tau_{y}(0)$ extracted from a fit to the $\tau(\dot{\gamma})$ data of a Herschel-Bulkley law of index $n=0.5$. Stars: dimensionless static yield stresses measured after a preshear at high shear rate (from Fig. 13). Empty circles: dimensionless static yield stress data from Mahaut et al. (2008a). Line: model (Eq. 31) based on the linear behavior observed by Thomas (1965) and Sierou and Brady (2002). Crosses: dimensionless consistency data plotted as $\left[\eta_{H B}(\phi) / \eta_{H B}(0)\right]^{1 /(n+1)}(1-\phi)^{n /(n+1)}$ with $n=0.5$.

shear.

We now report the values of the dimensionless static yield stresses measured in these experiments in Fig. 12, together with the dynamic yield stress, previous data of Mahaut et al. (2008a), and the predictions of the model. Of course, these static yield stresses are macroscopic values, possibly affected by migration effect and heterogeneities of the suspension microstructure; they provide nevertheless relevant estimates of the impact of microstructure on the behavior.

It is remarkable that the observed static yield stresses for materials presheared at high shear rate are now in much better agreement with the model (Eq. 31), and with the consistency data replotted as $\left[\eta_{H B}(\phi) / \eta_{H B}(0)\right]^{1 /(n+1)}(1-\phi)^{n /(n+1)}$ with $n=0.5$. In other words, there seems to be a link between the consistency values and the yield stress values as predicted by Eq. 23, provided the yield stress is measured for the microstructure formed at high shear, as is the consistency, which makes sense. This allows us to provide a full consistent picture of the behavior of the materials with the model presented in Sec. II. It also confirms that the shear-dependent function $g(\phi)$ bears sufficient information on the microstructure for each shear history as a first approximation.

It remains, however, that we have no prediction to make for the value of the dynamic yield stress, and we cannot compare its value to any other property of the material. This seems to be a specificity of suspensions in yield stress fluids. Indeed, in the spirit of the Chateau et al. (2008) and Mahaut et al. (2008a) works, Liard et al. (2014) have recently shown that the behavior of suspensions in power-law (shear-thinning and shear-thickening) fluids can be deduced for that of suspensions in Newtonian fluids. This is interpreted by the fact that distributions of large shear rates (those who bring the main contribution to dissipation) in the interstitial fluid are similar in both cases, i.e. that the microstructure formed under shear in these fluids share some similarities. At high shear rate, the behavior of yield stress suspensions should be expected to be similar to that of shear-thinning suspensions, and the same conclusions as in Liard et al. (2014) should apply. In this context, the agreement with the proposed model for the consistency values 
(Eq. 32) and for the static yield stress values (Eq. 31) comes from the fact that the $g(\phi)$ function we have chosen for the linear response, namely the Krieger-Dougherty law, is in good agreement with dimensionless viscosities of sheared suspensions of particles in Newtonian materials in the range of volume fractions investigated. However, the fact that the values of the dynamic yield stress cannot yet be predicted strongly suggests that the properties of shear in the interstitial fluid are different at the onset of plasticity, and that the approach made for power-law fluids cannot be fully generalized to the case of yield stress fluids. To characterize $g(\phi)$ for any shear history, a solution could consist in using parallel superposition (Vermant et al., 1998) or to stop abruptly the flow and to apply small oscillations to measure the suspension linear properties for the gelled microstructure; this cannot be done simply, however, in the case of inhomogeneous materials.

We also note in Fig. 12 that there is a good agreement between the model and the data of Mahaut et al. (2008a). This is surprising and seems to be fortuitous. Indeed, the static yield stress measurements of Mahaut et al. (2008a) where performed after loading into the rheometer, on isotropic (unsheared) materials (see Fig. 10c), and there is a priori no reason why materials with an isotropic microstructure and materials with the microstructure formed at high shear rate should have the same properties. The link between the microstructure and the rheological properties of yield stress suspensions has to be built to understand this puzzling observation.

\section{E Static yield stress vs dynamic yield stress: velocity profiles}

In order to confirm the above macroscopic observations at the local scale, we briefly investigate the impact of shear history on the flows of the material. To do that, we study the steady-state velocity profiles obtained with our rheo-NMR setup in a suspension in two cases, corresponding to the histories investigated in Fig. 11: (i) when the rotation velocity is slowly decreased from high to low velocities, and (ii) when flow is started after a preshear at high shear rate and a resting period. These velocity profiles are shown in Fig. 13.

At low rotational velocities $\Omega$, the profiles observed for these two histories are clearly different. When slowly decreasing $\Omega$, the velocity profiles have the shape classically observed in yield stress fluids (Coussot, 2005): the velocity decreases smoothly towards zero; when it reaches zero inside the gap, i.e. when there is shear localization, the slope of the velocity profile at the liquid/solid transition is zero (Figs. 13a,c). This is because this slope is the shear rate $\dot{\gamma}$, and because the shear stress value $\tau$ is equal to the yield stress at this transition: indeed, $\dot{\gamma} \rightarrow 0$ when $\tau \rightarrow \tau_{y}$ for a simple yield stress fluid. On the other hand, at start-up after a strong preshear, when the velocity profiles are localized, the velocity decreases abruptly towards zero, i.e. the slope of the profile at the liquid/solid transition is now different from zero (Figs. 13b,c). The shear rate in the flowing region is thus higher than a critical shear rate $\dot{\gamma}_{c}$, which is here of order $0.5 \mathrm{~s}^{-1}$. This is reminiscent of the behavior of thixotropic materials (Coussot, 2005; Ovarlez et al., 2013).

The interpretation of this behavior is sketched in Fig. 13d. After the preshear, the material has the microstructure formed under high shear, and its static yield stress $\tau_{y, s}$ is higher than the dynamic yield stress $\tau_{y, d}$. When flow starts at low $\Omega$, flow is localized and stops where the shear stress is equal to $\tau_{y, s}$. In the liquid region, flow reaches quickly a steady-state $\tau=\tau_{y, d}+\eta_{H B} \dot{\gamma}$. At the liquid/solid transition, in the flowing region, the shear stress is then $\tau=\tau_{y, d}+\eta_{H B} \dot{\gamma}_{c}$, which should equal $\tau_{y, s}$, since the material in the solid region has kept its initial microstructure. The difference between the shear history dependent static yield stress and the dynamic yield stress thus explains the existence of the critical shear rate $\dot{\gamma}_{c} \neq 0$ and of flow hysteresis.

To further illustrate the impact of shear history on the microstructure gelled at rest and on the material static yield stress, we have performed an additional experiment in which we have 
(a)

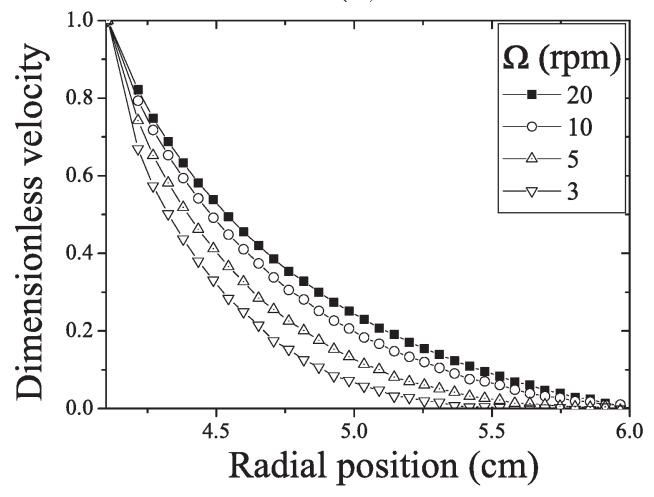

(c)

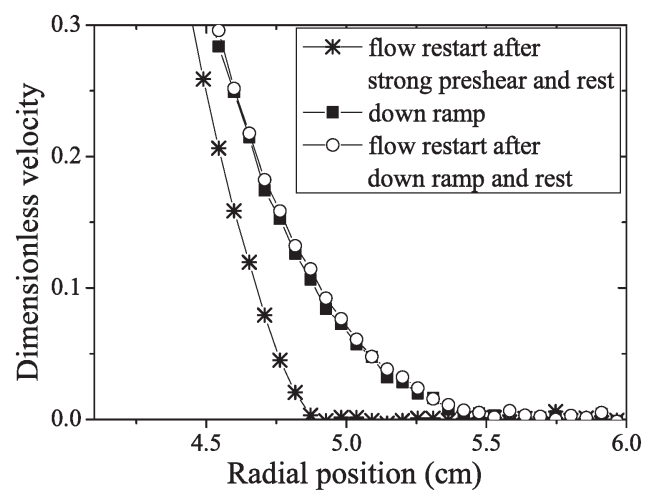

(b)

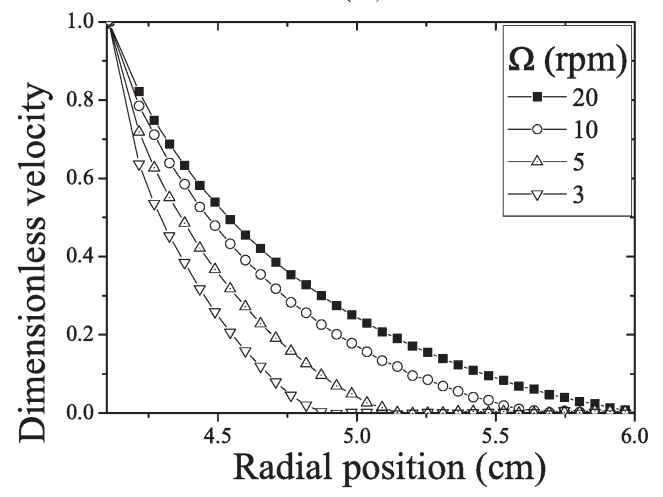

(d)

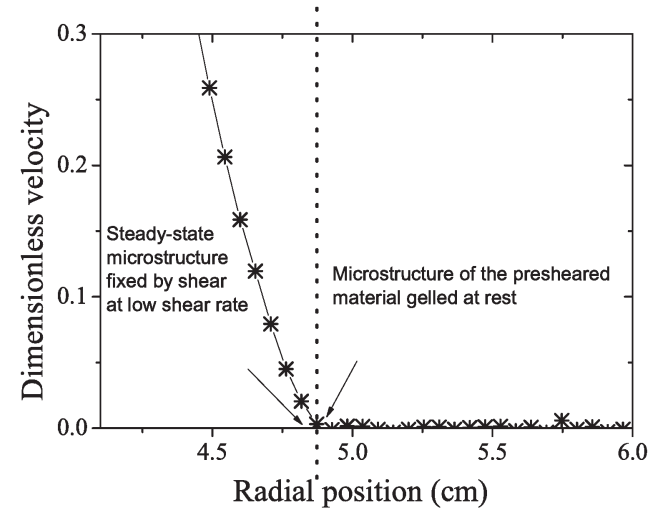

Figure 13: Velocity profiles in a suspension of volume fraction $\phi=40 \%$ in the rheo-NMR Couette geometry when velocity is slowly decreased from 100 to $3 \mathrm{rpm}$ (a) or measured at flow start at various velocities after a preshear at $100 \mathrm{rpm}$ and a 5 min resting period (b). (c) Focus on the behavior at $5 \mathrm{rpm}$ : role of shear history (see legend). (d) Sketch of the behavior at the liquid/solid transition for a flow restart after a strong preshear.

slowly ramped down the rotation velocity towards zero, left the material at rest to relax during 5 minutes, and restarted the flow at low rotational velocity. The resulting velocity profile, shown in Fig. 13c, is similar to that observed during the down ramp, which means that $\dot{\gamma}_{c}=0$ and that the static yield stress is equal to the dynamic yield stress for this shear history. This is consistent with the results of Secs. IVC and IVD: here, the microstructure is formed under low shear rate, when $\tau \simeq \tau_{y, d}$ before being gelled at rest; flow is thus restarted simply with $\tau_{y, s} \simeq \tau_{y, d}$.

To conclude on this point, we have confirmed here that a shear-history-dependent static yield stress emerges when adding particles in a yield stress fluid, due to the shear-history-dependent microstructure (Sec. IV C). In particular, a preshear at high shear rate results in a static yield stress much higher than the dynamic yield stress. At the local scale, this induces flow hysteresis. 
(a)

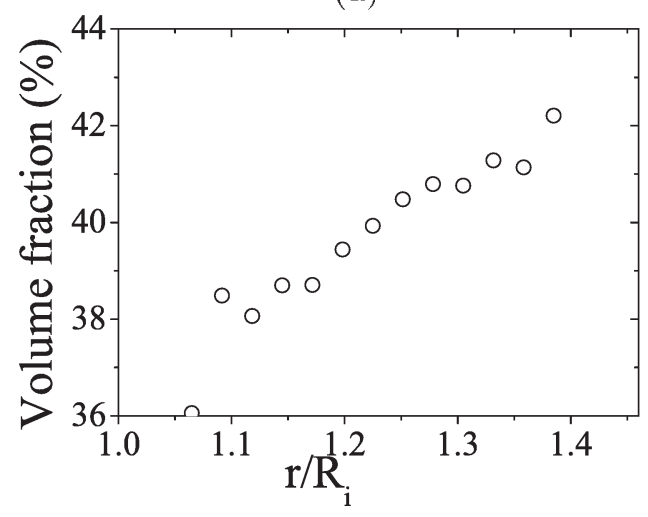

(c)

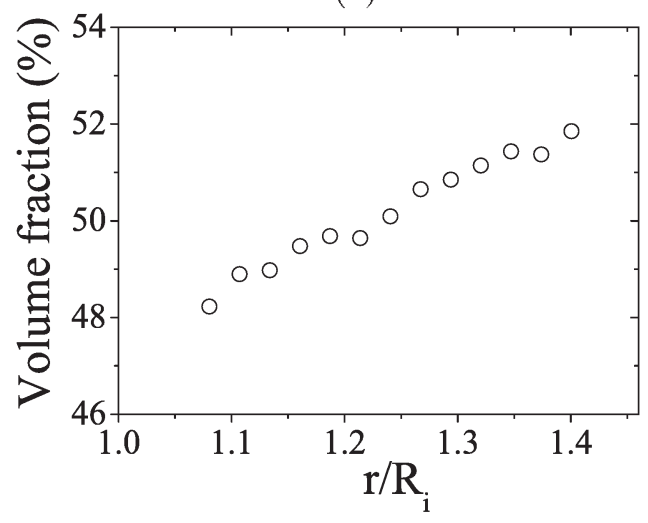

(b)

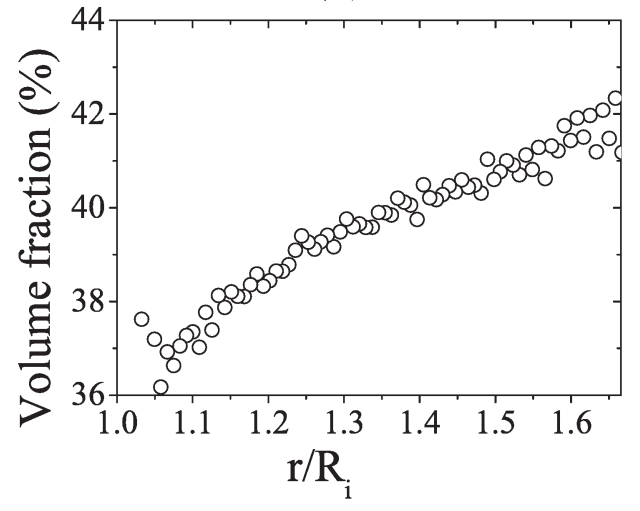

(d)

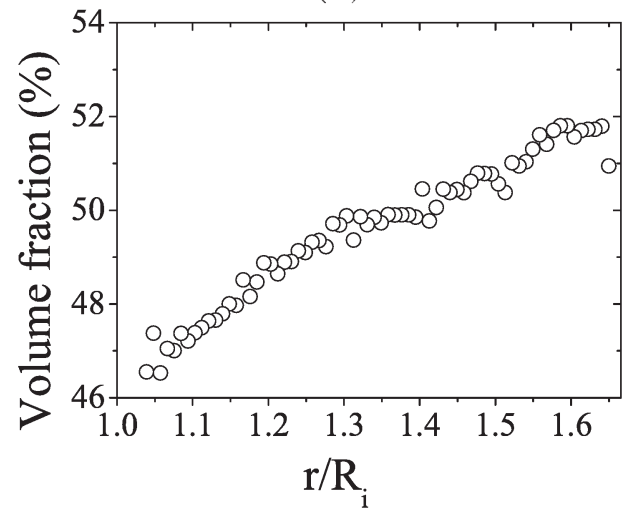

Figure 14: Steady volume fraction profiles versus dimensionless radial position $r / R_{i}$ : in suspensions of $40 \%$ (a) and $50 \%$ (c) particles in a concentrated emulsion; in suspensions of $40 \%$ (b) and $50 \%$ (d) particles in a Newtonian oil. For the yield stress suspensions, the inner cylinder radius is $R_{i}=4.1 \mathrm{~cm}$, and the gap is $1.9 \mathrm{~cm}$ wide; for the viscous suspensions, $R_{i}=3 \mathrm{~cm}$, and the gap is $2 \mathrm{~cm}$ wide.

\section{F Volume fraction profiles and normal stresses}

We finally comment briefly on the volume fraction profiles. One of the predictions of the model presented in Sec. II is that steady volume fraction profiles in a yield stress fluid, in the absence of shear localization, should be similar to those observed in a Newtonian fluid. To test this prediction, we have performed experiments with suspensions of monodisperse spherical particles in a Newtonian oil (polyethylene glycol, as in Boyer et al. (2011b)), in which steady volume fraction profiles were measured; note that the dimensionless viscosity $g(\phi)$ of this system is better fitted by the Zarraga equation (Eq. 30) than by the Krieger-Dougherty equation (Boyer et al., 2011b), which implies that perfect match between experiments performed with the Newtonian interstitial fluid and with the yield stress fluid cannot be expected. Due to a change of the rheo-NMR setup, these experiments could not be performed in the exact same geometries: the concentric-cylinder Couette cell used to study the viscous suspensions is of inner cylinder radius $R_{i}=3 \mathrm{~cm}$ and of gap size $2 \mathrm{~cm}$. Nevertheless, these dimensions are comparable to those of the Couette cell used to study the yield stress suspensions, which allows us to compare the profiles. 
(a)

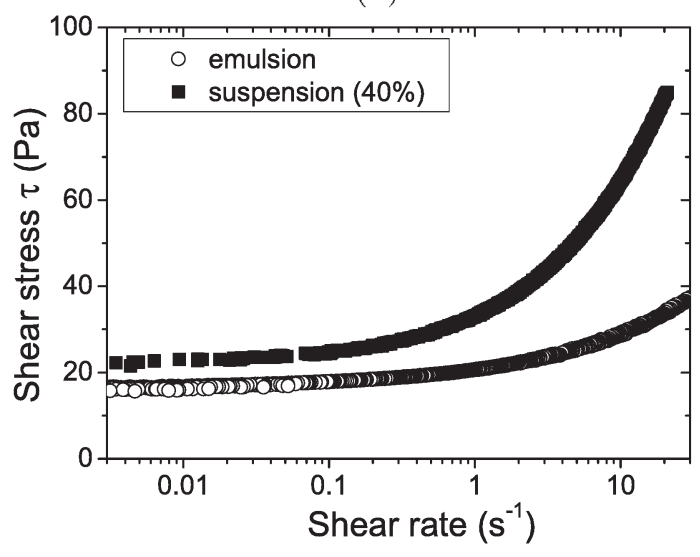

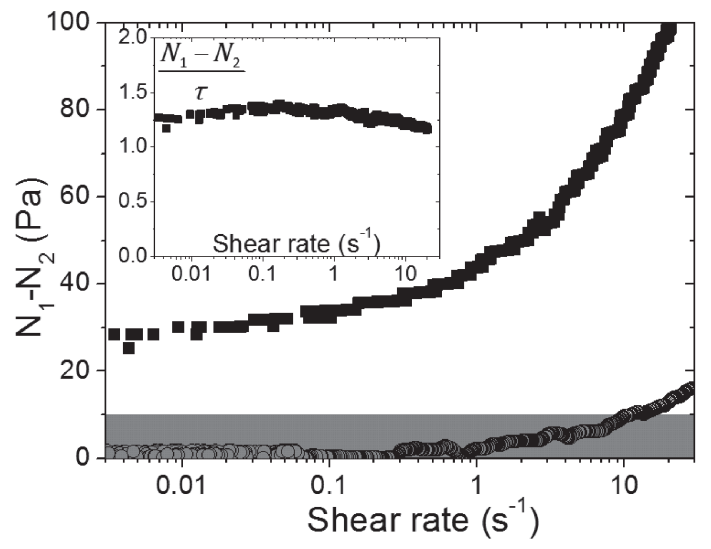

Figure 15: Shear stress $\tau$ (a) and combination of normal stress differences $N_{1}-N_{2}$ (b) vs. shear rate $\dot{\gamma}$, measured in a parallel plate geometry in an emulsion (open circles) and in the same emulsion filled with $40 \%$ of particles (squares). The grey zone shows the range of possible tension surface effects. The inset of Fig. 15b shows the ratio $\left(N_{1}-N_{2}\right) / \tau$ vs. $\dot{\gamma}$ in the suspension.

The steady volume fraction profiles obtained in the two fluids are shown in Fig. 14 for two particle volume fractions: $40 \%$ and $50 \%$. It is observed that these profiles have the same shape in both fluids, and are characterized by the same amplitude of volume fraction inhomogeneities. There is thus no noticeable difference between steady-state migration in a yield stress fluid and in a Newtonian fluid, as long as there is no plug zone in the case of the yield stress suspensions; of course, things should be different for the kinetics of the phenomenon, which was not studied here, as it can be expected to have a complex dependence on the shear rate in a nonlinear fluid.

As explained in detail in Sec. II C, this independence of the steady volume fraction profiles on the fluid properties can be predicted from the assumption that the ratio of the particle normal stress to the shear stress should be a function of $\phi$ only, as in a Newtonian fluid, and that this function should not be affected by the nature of the fluid. To illustrate this point further, we show the combination of normal stress differences $N_{1}-N_{2}$ measured in a parallel plate geometry for a pure emulsion, and for the same emulsion filled with $40 \%$ of particles. The data are shown in Fig. 15.

Before analyzing the data, it should be noted that normal stresses data are affected by surface tension effects at the edges of parallel plate geometries (Hutton, 1972). This is particularly hard to take into account for the yield stress fluids we study since the shape of the free surface is seen to change with the shear rate. Assuming that the emulsion/air surface tension is the same as the continuous phase (dodecane)/air surface tension $(25 \mathrm{mN} / \mathrm{m})$, we can estimate that there is a $10 \mathrm{~Pa}$ uncertainty on normal stress differences due to changes in the shape of the free surface.

In Fig. 15, it is first observed that the flows of the concentrated emulsion seem to be characterized themselves by a combination of normal stress differences $N_{1}-N_{2}$ which tends towards zero at low shear rate as expected theoretically (Seth et al., 2011). Nevertheless, in the range of shear rates investigated, it is hard to say what is the true value of $N_{1}-N_{2}$ because of the shear-rate-dependent contribution of surface tension due to free surface shape changes. The normal stress differences can nevertheless be expected to be relatively important at high shear rates $\left(\geq 100 \mathrm{~s}^{-1}\right)$. They can thus be expected to play a role on the rheology of the suspensions at high shear rate and at high $\phi$ (where the shear rate in the interstitial fluid is highly magnified), 
which remains to be investigated theoretically (we recall that the theory developed in Sec. II is valid for an isotropic interstitial fluid). At this stage, we leave this point apart, and focus on the suspension behavior.

It is particularly striking that, in the suspension, $N_{1}-N_{2}$ is characterized by a nonzero critical value $\left[N_{1}-N_{2}\right]_{y}$, which is its limit value as $\dot{\gamma}$ tends toward zero (given its value, it cannot be an artefact due to surface tension). This feature, absent from the interstitial fluid, is in agreement with the theory (see Eq. 25), and comes from the fact that normal stress differences are assumed to be proportional to the apparent interstitial viscosity, as in viscous suspensions. To further check this point, we plot the ratio of $N_{1}-N_{2}$ to the shear stress $\tau$ in Fig. 15c. We observe, as predicted by the theory, and in line with the observations on shear-induced migration, that this ratio does not depend much on the shear rate. It is here of order 1.2. For suspensions of $40 \%$ particles in a Newtonian fluid, it was observed to be of order 0.3 by Dai et al. (2013) and of order 0.5 by Dbouk et al. (2013). We cannot comment further this difference, a detailed study of normal stress differences, with appropriate correction of shear-rate-dependent surface tension effects, being out of the scope of the present paper.

We finally note that some conclusions of this part, namely the validity of Eq. 36 and the existence of a yield particle normal stress, are supported by a recent paper, published while our paper was under review, where shear stress and particle normal stresses have been studied in detail for $\phi_{\text {div }}>\phi>45 \%$ (Dagois-Bohy et al., 2015). A notable result from this work is that the apparent friction coefficient of the yield stress suspensions follows the same law as a function of $\phi$ as in viscous suspensions.

\section{Conclusion}

The main findings of our work are the following:

- We have shown that the steady flow behavior of the suspensions can be modeled by a Herschel-Bulkley behavior of same index as the interstitial fluid, which is predicted by the theory, in which the only source of nonlinearity of the behavior is in the interstitial fluid. This should be strictly true only if the particle microstructure does not show significant variations with the shear rate at high shear rate; the yield stress has no importance for this property: this should hold for power-law materials. Note that our experiments are not sensitive to small variations (smaller than, say, 0.1) of the index $n$. Finer investigations might thus be useful to strengthen or invalidate this point.

- We have observed a good agreement between the increase of the consistency with the particle volume fraction in the 0-50\% range and the theoretical prediction (Eq. 32) based on the works of Chateau et al. (2008) and Mahaut et al. (2008a). The similarity between the function $g(\phi)$ used to model the behavior of the isotropic suspensions studied by Mahaut et al. (2008a) and that used to model the suspension behavior under steady shear at high shear rate remains to be understood, the microstructures being significantly different. The fact that this function $g(\phi)$ is the same as for some viscous suspensions is however consistent with the conclusions of Liard et al. (2014) for power-law fluids. The similarity with the Sierou and Brady (2002) simulations suggests that the particles behave as low friction particles; this might be due to the large size of the constitutive elements of the yield stress fluid as compared to the particle roughness and to their high concentration, which may hinder frictional interactions. 
- We have observed shear-induced migration with similar properties as in a Newtonian fluid, which we have predicted theoretically. This suggests that particle normal stresses are proportional to the shear stress as for suspensions of particles in Newtonian fluids; this is a strong validation of the theoretical approach, which consists in linearizing the interstitial fluid behavior: in this approach, a change of the apparent viscosity of the interstitial material (due to a change of the shear rate) impacts similarly all components of the suspension stress tensor.

- In line with this last observation, we have observed the emergence of a nonzero normal stress difference at the yielding transition, which is absent of the interstitial fluid behavior. This is a signature of the fact that the rheology is governed by the apparent viscosity of the sheared interstitial material.

- Thanks to an original rheo-X-ray microtomography setup, we have shown that the steadystate suspension microstructure (the particle spatial distribution) in a yield stress fluid depends on the shear rate, which contrasts with the case of a Newtonian interstitial fluid. This leads to an original shear-dependent macroscopic behavior. In particular, whereas the emulsion is a simple yield stress fluid, the yield stress of the suspensions at flow start can differ significantly from the yield stress at flow stoppage, depending on flow history. We showed that the static yield stress is the yield stress of the material which has the microstructure formed during its preparation, whereas the dynamic yield stress is the yield stress of the material whose microstructure is the steady-state microstructure formed under low shear. The existence of a shear-rate dependent microstructure also explains the discrepancy between the theory prediction for the consistency and that for the yield stress at flow stoppage, since a unique microstructure is assumed in the theory. Further progresses in the modeling of suspensions of particles in yield stress fluids would imply to model the dependence of microstructure on the shear rate, and to take it into account in the modeling of the macroscopic properties. It remains that, at this stage, we are unable to predict the value of the dynamic yield stress of the suspensions.

\section{Acknowledgments}

This work was supported by a grant from Agence National de la Recherche (ANR 2010 JCJC 090501 - SUSPASEUIL). The Laboratoire Navier microtomograph has been acquired with the financial support of Région Île-de-France.

\section{References}

Abbott, J. R., N. Tetlow, A. L. Graham, S. A. Altobelli, E. Fukushima, L. A. Mondy, and T. S. Stephens, "Experimental observations of particle migration in concentrated suspensions: Couette flow," J. Rheol. 35, 773-795 (1991).

Acrivos, A., "BINGHAM AWARD LECTURE-1994 Shear-induced particle diffusion in concentrated suspensions of noncolloidal particles," J. Rheol. 39, 813-826 (1995).

Altobelli, S. A., R. C. Givler, and E. Fukushima, "Velocity and concentration measurements of suspensions by nuclear magnetic resonance imaging," J. Rheol. 35, 721-734 (1991). 
Altobelli, S. A., E. Fukushima, and L. A. Mondy, "Nuclear magnetic resonance imaging of particle migration in suspensions undergoing extrusion," J. Rheol. 41, 1105-1115 (1997).

Ancey, C., and H. Jorrot, "Yield stress for particle suspensions within a clay dispersion," J. Rheol. 45, 297-319 (2001).

Beris, A. N., J. A. Tsamopoulos, R. C. Armstrong, and R. A. Brown, "Creeping motion of a sphere through a Bingham plastic," J. Fluid Mech. 158, 219-244 (1985).

Blanc, F., F. Peters, and E. Lemaire, "Experimental signature of the pair trajectories of rough spheres in the shear-induced microstructure in noncolloidal suspensions," Phys. Rev. Lett. 107, 208302 (2011a).

Blanc, F., F. Peters, and E. Lemaire, "Local transient rheological behavior of concentrated suspensions," J. Rheol. 55, 835-854 (2011b).

Blanc, F., E. Lemaire, A. Meunier, and F. Peters, "Microstructure in sheared non-Brownian concentrated suspensions," J. Rheol. 57, 273-292 (2013).

Bonnoit, C., T. Darnige, E. Clement, and A. Lindner, "Inclined plane rheometry of a dense granular suspension," J. Rheol. 54, 65-79 (2010).

Boyer, F., O. Pouliquen, and E. Guazzelli, "Dense suspensions in rotating-rod flows: Normal stresses and particle migration," J. Fluid Mech. 686, 5-25 (2011a).

Boyer, F., E. Guazzelli, and O. Pouliquen, "Unifying suspension and granular rheology," Phys. Rev. Lett. 107, 188301 (2011b).

Brady, J. F., and J. F. Morris, "Microstructure of strongly sheared suspensions, and its impact on rheology and diffusion," J. Fluid Mech. 348, 103-139 (1997).

Callaghan, P. T., Principles of Nuclear Magnetic Resonance Spectroscopy (Clarendon, Oxford, 1991).

Callaghan, P. T. (1999), "Rheo-NMR: nuclear magnetic resonance and the rheology of complex fluids," Rep. Prog. Phys. 62, 599-670 (1999).

Chateau, X., G. Ovarlez, and K. L. Trung, "Homogenization approach to the behavior of suspensions of noncolloidal particles in yield stress fluids," J. Rheol. 52, 489-506 (2008).

Cheddadi, I., P. Saramito, and F. Graner, "Steady couette flows of elastoviscoplastic fluids are nonunique," J. Rheol. 56, 213-239 (2012).

Coussot, P., Mudflow Rheology and Dynamics (Balkema, Rotterdam, 1997).

Coussot, P., Rheometry of Pastes, Suspensions and Granular Materials (John Wiley \& Sons, Hoboken, 2005).

Couturier, E., F. Boyer, O. Pouliquen, and E. Guazzelli, Suspensions in a tilted trough: Second normal stress difference," J. Fluid Mech. 686, 26-39 (2011).

Dagois-Bohy, S., S. Hormozi, E. Guazzelli, and O. Pouliquen, "Rheology of dense suspensions of non-colloidal spheres in yield-stress fluids," J. Fluid Mech. 776, R2 (2015). 
Dai, S.-C., E. Bertevas, F. Qi, and R. I. Tanner, "Viscometric functions for noncolloidal sphere suspensions with Newtonian matrices," J. Rheol. 57, 493-510 (2013).

Dbouk, T., L. Lobry, and E. Lemaire, "Normal stresses in concentrated non-Brownian suspensions," J. Fluid Mech. 715, 239-272 (2013).

Deboeuf, S., N. Lenoir, D. Hautemayou, C. Mézière, and G. Ovarlez, "Microstructure and Rheology of Particle Suspension in a Yield Stress Fluid," in Tomography of Materials and Structures, International Conference on Tomography of Materials and Structures, Gand, Belgium, 2013.

Denn, M. M., and J. F. Morris, "Rheology of non-Brownian suspensions," Annu. Rev. Chem. Biomol. Eng. 5, 203-228 (2014).

Dzuy, N. Q., and D. V. Boger, "Yield stress measurement for concentrated suspensions," J. Rheol. 27, 321-349 (1983).

Erdogan, T.S., " Determination of aggregate shape properties using X-ray tomographic methods and the effect of shape on concrete rheology," PhD thesis, University of Texas at Austin, 2005.

Fall, A., A. Lemaitre, F. Bertrand, D. Bonn, and G. Ovarlez, "Shear thickening and migration in granular suspensions," Phys. Rev. Lett. 105, 268303 (2010).

Fall, A., F. Bertrand, D. Hautemayou, C. Mezière, P. Moucheront, A. Lemaître, and G. Ovarlez, "Macroscopic discontinuous shear thickening versus local shear jamming in cornstarch," Phys. Rev. Lett. 114, 098301 (2015).

Frankel, N. A., and A. Acrivos, "On the Viscosity of a Concentrated Suspension of Solid Spheres," Chem. Eng. Sci. 22, 847-852 (1967).

Gallier, S., E. Lemaire, F. Peters, and L. Lobry, "Rheology of sheared suspensions of rough frictional particles," J. Fluid Mech. 757, 514-549 (2014).

Garland, S., G. Gauthier, J. Martin, and J. F. Morris, "Normal stress measurements in sheared non-Brownian suspensions," J. Rheol. 57, 71-88 (2013).

Geiker, M. R., M. Brandl, L. N. Thrane, and L. F. Nielsen, "On the effect of coarse aggregate fraction and shape on the rheological properties of self-compacting concrete," Cem. Concr. Aggreg. 24, 3-6 (2002).

Hafid, H., G. Ovarlez, F. Toussaint, P. H. Jezequel, and N. Roussel, "Assessment of potential concrete and mortar rheometry artifacts using magnetic resonance imaging," Cem. Concr. Res. 71, 29-35 (2015).

Hutton, J. F., "Effect of changes of surface tension and contact angle on normal force measurement with the Weissenberg rheogoniometer," Rheol. Acta 11, 70-72 (1972).

Jau, W. C., and C. T. Yang, "Development of a modified concrete rheometer to measure the rheological behavior of conventional and self-consolidating concretes," Cem. Concr. Compos. 32, 450-460 (2010).

Joumana, Y.M., M. Chaouche, M. Guerinet, M. Moranville, and N. Roussel, "From ordinary rhelogy concrete to self compacting concrete: a transition between frictional and hydrodynamic interactions," Cem. Concr. Res. 38, 890-896 (2008). 
Keentok, M., "The measurement of the yield stress of liquids," Rheol. Acta 21, 325-332 (1982)

Koehler E. P., D. W. Fowler, C. F. Ferraris, and S. A. Amziane, "New, portable rheometer for fresh self-consolidating concrete," ACI Mater. J. 233, 97-116 (2006).

Leighton, D., and A. Acrivos, "Measurement of shear-induced self-diffusion in concentrated suspensions of spheres," J. Fluid Mech. 177, 109-131 (1987a).

Leighton, D., and A. Acrivos, "The shear-induced migration of particles in concentrated suspensions," J. Fluid Mech. 181, 415-439 (1987b).

Lhuillier, D., "Migration of rigid particles in non-brownian viscous suspensions," Phys. Fluids 21, 023302 (2009).

Liard, M., N. S. Martys, W. L. George, D. Lootens, and P. Hebraud, "Scaling laws for the flow of generalized Newtonian suspensions," J. Rheol. 58, 1993-2015 (2014).

Maire, E., J. Y. Buffiere, L. Salvo, J. J. Blandin, W. Ludwig, and J. M. Letang, "On the application of X-ray microtomography in the field of materials science," Adv. Eng. Mater. 3, 539-546 (2001).

Mahaut, F., X. Chateau, P. Coussot, and G. Ovarlez, "Yield stress and elastic modulus of suspensions of noncolloidal particles in yield stress fluids," J. Rheol. 52, 287-313 (2008a).

Mahaut, F., S. Mokeddem, X. Chateau, N. Roussel, and G. Ovarlez, "Effect of coarse particle volume fraction on the yield stress and thixotropy of cementitious materials," Cem. Concr. Res. 38, 1276-1285 (2008b).

Martínez-Padilla, L. P., and C. Rivera-Vargas, "Flow behavior of Mexican sauces using a vanein-a-large cup rheometer," J. Food Eng. 72, 189-196 (2006).

Mason, T. G., J. Bibette, and D. A. Weitz, "Yielding and flow of monodisperse emulsions," J. Colloid Interface Sci. 179, 439-448 (1996).

Mewis, J., and N. Wagner, Colloidal suspension rheology (Cambridge University Press, Cambridge, 2012).

Mills, P., and P. Snabre, "Rheology and Structure of Concentrated Suspensions of Hard Spheres. Shear Induced Particle Migration," J. Phys. II France 5, 1597-1608 (1995).

Mohan, L., M. Cloitre, and R. T. Bonnecaze, "Build-up and two-step relaxation of internal stress in jammed suspensions," J. Rheol. 59, 63-84 (2015).

Morris, J. F., and F. Boulay, "Curvilinear flows of noncolloidal suspensions: The role of normal stresses," J. Rheol. 43, 1213-1237 (1999).

Morris, J., "A review of microstructure in concentrated suspensions and its implications for rheology and bulk flow," Rheol. Acta 48, 909-923 (2009).

Narumi, T., H. See, Y. Honma, T. Hasegawa, T. Takahashi, and N. Phan-Thien, "Transient response of concentrated suspensions after shear reversal," J. Rheol. 46, 295-305 (2002).

Nott, P., and J. F. Brady, "Pressure-driven flow of suspensions: Simulation and theory," J. Fluid Mech. 275, 157-199 (1994). 
Nott, P., E. Guazzelli, and O. Pouliquen, "The suspension balance model revisited," Phys. Fluids 23, 043304 (2011).

Ovarlez, G., F. Bertrand, and S. Rodts, "Local determination of the constitutive law of a dense suspension of noncolloidal particles through magnetic resonance imaging," J. Rheol. 50, 259$292(2006)$.

Ovarlez, G., S. Rodts, A. Ragouilliaux, P. Coussot, J. Goyon, and A. Colin, Wide-gap Couette flows of dense emulsions: Local concentration measurements, and comparison between macroscopic and local constitutive law measurements through magnetic resonance imaging," Phys. Rev. E 78, 036307 (2008).

Ovarlez, G., Q. Barral, and P. Coussot, "Three-dimensional jamming and flows of soft glassy materials," Nature Mater. 9, 115-119 (2010).

Ovarlez, G., F. Mahaut, F. Bertrand, and X. Chateau, "Flows and heterogeneities with a vane tool: Magnetic resonance imaging measurements," J. Rheol. 55, 197-223 (2011).

Ovarlez, G., F. Bertrand, P. Coussot, and X. Chateau, "Shear-induced sedimentation in yield stress fluids," J. Non-Newt. Fluid Mech. 177, 19-28 (2012).

Ovarlez, G., S. Cohen-Addad, K. Krishan, J. Goyon, and P. Coussot, "On the existence of a simple yield stress fluid behavior," J. Non-Newt. Fluid Mech. 193, 68-79 (2013).

Parsi, F., and F. Gadala-Maria, "Fore-and-aft asymmetry in a concentrated suspension of solid spheres," J. Rheol. 31, 725-732 (1987).

Phillips, R. J., R. C. Armstrong, R. A. Brown, A. L. Graham, and J. R. Abbott, "A constitutive equation for concentrated suspensions that accounts for shear-induced particle migration," Phys. Fluids A: Fluid Dynamics 4, 30-40 (1992).

Raynaud, J. S., P. Moucheront, J. C. Baudez, F. Bertrand, J. P. Guilbaud, and P. Coussot, "Direct determination by NMR of the thixotropic and yielding behavior of suspensions," J. Rheol. 46, 709-732 (2002).

Rodts, S., F. Bertrand, S. Jarny, P. Poullain, and P. Moucheront, "Développements récents dans l'application de l'IRM à la rhéologie et à la mécanique des fluides," C. R. Chim. 7, 275-282 (2004).

Rodts, S., J. Boujlel, B. Rabideau, G. Ovarlez, N. Roussel, P. Moucheront, and P. Coussot, "Solid-liquid transition and rejuvenation similarities in complex flows of thixotropic materials studied by NMR and MRI," Phys. Rev. E 81, 021402 (2010).

Saak, A. W., H. M. Jennings, and S. P. Shah, "The influence of wall slip on yield stress and viscoelastic measurements of cement paste," Cem. Concr. Res. 31, 205-212 (2001).

Sengun, M. Z., and R. F. Probstein, "Bimodal model of slurry viscosity with application to coal-slurries. Part 1. Theory and experiment," Rheol. Acta 28 382-393 (1989).

Sengun, M. Z., and R. F. Probstein, "Bimodal model of slurry viscosity with application to coal-slurries. Part 2. High shear limit behavior," Rheol. Acta 28 394-401 (1989). 
Seth, J. R., L. Mohan, C. Locatelli-Champagne, M. Cloitre, and R. T. Bonnecaze, "A micromechanical model to predict the flow of soft particle glasses," Nature Mater. 10, 838-843 (2011).

Shapley, N. C., R. A. Brown, and R. C. Armstrong, "Evaluation of particle migration models based on laser Doppler velocimetry measurements in concentrated suspensions," J. Rheol. 48, 255-279 (2004).

Sierou, A., and J. F. Brady, "Rheology and microstructure in concentrated noncolloidal suspensions," J. Rheol. 46, 1031-1056 (2002).

Stickel, J., and R. Powell, "Fluid mechanics and rheology of dense suspensions," Ann. Rev. Fluid Mech. 37, 129-149 (2005).

Suquet, P., editor, Continuum Micromechanics (Springer-Verlag, Wien, New York, 1997).

Tabuteau, H., P. Coussot, and J. R. De Bruyn, "Drag force on a sphere in steady motion through a yield-stress fluid," J. Rheol. 51, 125-137 (2007).

Tetlow, N., A. L. Graham, M. S. Ingber, S. R. Subia, L. A. Mondy, and S. A. Altobelli, "Particle migration in a Couette apparatus: Experiment and modeling," J. Rheol. 42, 307-327 (1998).

Thomas, D. G., "Transport characteristics of suspension: VIII. A note on the viscosity of newtonian suspensions of uniform spherical particles," J. Colloid Sci. 20, 267-277 (1965).

Torquato, S., Random heterogeneous materials: microstructure and macroscopic properties (Springer Science \& Business Media, Heidelberg, 2002).

Toutou, Z., and N. Roussel, Multi scale experimental study of concrete rheology: From water scale to gravel scale," Mater. Struct. 39, 189-199 (2006).

Vermant, J., L. Walker, P. Moldenaers, and J. Mewis, "Orthogonal versus parallel superposition measurements," J. Non-Newt. Fluid Mech. 79, 173-189 (1998).

Vu, T.-S., G. Ovarlez, and X. Chateau, "Macroscopic behavior of bidisperse suspensions of noncolloidal particles in yield stress fluids," J. Rheol. 54, 815-833 (2010).

Zarraga, I. E., D. A. Hill, and D. T. Leighton, "The characterization of the total stress of concentrated suspensions of noncolloidal spheres in Newtonian fluids," J. Rheol. 44, 185-220 (2000). 